Modern Physics Letters B

Vol. 31, No. 36 (2017) 1799001 (37 pages)

(c) World Scientific Publishing Company

DOI: $10.1142 / \mathrm{S} 0217984917990019$

\title{
AUTHOR INDEX Volume 31
}

Abbas, H., see Javed

Abdel-Rahman, M., Alduraibi, M., Zia, M.F., Bahidra, E. \& Alasaad, A., Vanadium sesquioxide $\left(\mathrm{V}_{2} \mathrm{O}_{3}\right)$-based semiconducting temperature sensitive resistors for uncooled microbolometers

Abdel-Rahman, M., Salah, M., Ibrahim, A.M. \& Badawi, E.A., Comparative techniques to investigate plastically deformed 5754 Al-alloy

Abedi Ravan, B., see Ansarino

Abedi-Varaki, M., Study of carbon dioxide gas treatment based on equations of kinetics in plasma discharge reactor

Abou-Aly, A.I., see Anas

Abu Hassan, H., see Kadhim

Acar, S., see Karaduman

Achkir, O., Daoud, M. \& Mansour, M., Generalized graph states and mutually unbiased bases from multiqudits phase states

Adlan, W., see Ibraheem

Ahmadi, M., see Rouhi

Ahmadzadeh, M., see Kordani

Ahmed, R.M. \& Morsi, R.M.M., Polymer nanocomposite dielectric and electrical properties with quantum dots nanofiller

Akel, M., Sh. Ismael, Lee, S., Saw, S.H. \& Kunze, H.J.,

\author{
B31 (2017) 1750020 \\ B31 (2017) 1750145
}

B31 (2017) 1750255

B31 (2017) 1750010

B31 (2017) 1750210

B31 (2017) 1750290

B31 (2017) 1750114

B31 (2017) 1750284

B31 (2017) 1750183

B31 (2017) 1750068

B31 (2017) 1750053

B31 (2017) 1750235

B31 (2017) 1750278
Numerical experiments on the PF1000 plasma focus device operated with nitrogen and oxygen gases

Akgol, O., Karaaslan, M., Unal, E. \& Sabah, C., Implementation of a perfect metamaterial absorber into multi-functional sensor applications

Akgol, O., see Altintas

Al-Jawad, S.M.H., Rafic, S.N. \& Muhsen, M.M., Preparation and characterization of polyanilinecadmium sulfide nanocomposite for gas sensor application

Alaboz, H., see Nebioğlu

Alakbarov, O., see Samadov

Alasaad, A., see AbdelRahman

Alazba, A.A., see Amin

Alduraibi, M., see AbdelRahman

Aliyu, A.I., see Inc

Alizadeh, M., see Kordani

Altan, H., see Nebioğlu

Altan, H., see Shawky

Altintas, O., Unal, E., Akgol, O., Karaaslan, M., Karadag, F. \& Sabah, C., Design of a wide band metasurface as a linear to circular polarization converter

Amin, M.T. \& Alazba, A.A., Structural study of monoclinic $\mathrm{TiO}_{2}$ nanostructures and photocatalytic applications for degradation of
B31 (2017) 1750167

B31 (2017) 1750176 B31 (2017) 1750274

B31 (2017) 1750234

B31 (2017) 1750074

B31 (2017) 1750134

B31 (2017) 1750145

B31 (2017) 1750264

B31 (2017) 1750145

B31 (2017) 1750163

B31 (2017) 1750235

B31 (2017) 1750074

B31 (2017) 1750072

B31 (2017) 1750274 
crystal violet dye

Amolo, G.O., see Ibraheem

An, C., see $\mathrm{Li}$

An, L., see Yuan

Anas, M., Ebrahim, S., Eldeen, I.G., Awad, R. \& Abou-Aly, A.I., Dielectric properties of (SWCNTS) $x$ $\mathrm{GdBa}_{2} \mathrm{CuO}_{7-\delta}$ superconductor nanocomposites

Andreev, P.A., Simultaneous dipole and quadrupole moment contribution in the Bogoliubov spectrum: Application of the nonintegral Gross-Pitaevskii equation

Ansari, R., see Rouhi

Ansari, R., see Rouhi

Ansarino, M. \& Abedi Ravan, B., First-principles calculations of perpendicular magnetic anisotropy for spintronic applications

Ao, P., see Leng

Apostolov, A.T., Apostolova, I.N. \& Wesselinowa, J.M., Influence of spin-phonon interactions and spinreorientation transitions on the phonon properties of $R \mathrm{CrO}_{3}$

Apostolov, A.T., Apostolova, I.N., Trimper, S. \& Wesselinowa, J.M., Room temperature ferromagnetism in pure and ion-doped $\mathrm{SnO}_{2}$ nanoparticles

Apostolova, I.N., see Apostolov

Apostolova, I.N., see Apostolov

Arbab, A.I., Extended electrodynamics and its consequences

Ardashev, D.V., see Kuai

Asadpour, S.H., see Gh. Solookinejad

Asif, A., see Asif

Asif, A., see Asif

Asif, M. \& Asif, A., Effects of internal inductance on the Shafranov parameter by using the solution of equilibrium problem

\begin{abstract}
B31 (2017) 1750264
B31 (2017) 1750068

B31 (2017) 1750004

B31 (2017) 1750045
\end{abstract}

B31 (2017) 1750290

B31 (2017) 1750152

B31 (2017) 1750208

B31 (2017) 1750053

B31 (2017) 1750010 B31 (2017) 1740053

B31 (2017) 1750009

B31 (2017) 1750351

B31 (2017) 1750351

B31 (2017) 1750009

B31 (2017) 1750099

B31 (2017) 1750025

B31 (2017) 1750322

B31 (2017) 1750196

B31 (2017) 1750078

B31 (2017) 1750078
Asif, M. \& Asif, A., Theoretical calculation of electron density and temperature in the edge of tokamak

B31 (2017) 1750196

B31 (2017) 1750092

B31 (2017) 1750290

B31 (2017) 1750313

Azrina, A., see Mahyuddin

Badawi, E.A., see AbdelRahman

Bagheri, M., see Karimipour

Baghshahi, H.R., see Faraji

Baharvandi, H.R., see Kordani

Bahidra, E., see AbdelRahman

Bai, J., see $\mathrm{He}$

Bai, M., see Zhou

Balsera, I.T., see Song

Bao, X., see Wang

Bao, Y., see Liang

Baqi, S., see Javed

Belonenko, M., see Konobeeva

Belonenko, M.B., see Konobeeva

B31 (2017) 1750255

B31 (2017) 1750297

B31 (2017) 1750038

B31 (2017) 1750235

B31 (2017) 1750145

B31 (2017) 1750095

B31 (2017) 1750190

B31 (2017) 1750160

B31 (2017) 1750193

B31 (2017) 1740059

B31 (2017) 1750020

B31 (2017) 1750340

B31 (2017) 1750005

Benlattar, M., Elkoraychy, E., Sbiaai, K., Mazroui, M. \& Boughaleb, Y., EhrlichSchwöbel barriers and adsorption of $\mathrm{Au}, \mathrm{Cu}$ and $\mathrm{Ag}$ stepped (100) surfaces

Bentaiba, M., see Mazouz

Bhatt, S., Kumar, R. \& Kumar, M., Specific heat and thermal conductivity of nanomaterials

Bi, H., see Jiang

Bi, K., see Wang

Bi, K., see Zhang

Bi, L., Wu, W., Zhang, J. \& Yang, H., An assembly method for micro parts jointing with given space angle based on projection matching

Bi, W.Y., Li, L.H., Liu, H.T. \& Zhao, G., The effect of biased plates on transport of vacuum arc plasma through rectangular curved magnetic filter

Bian, X., Miao, M., Li, Z. \& Cui, X., U-shaped meander-line slow-wave structure with stub-loading
B31 (2017) 1750037

B31 (2017) 1750019

B31 (2017) 1750011

B31 (2017) 1740004

B31 (2017) 1750354

B31 (2017) 1750222

B31 (2017) 1750041

B31 (2017) 1740006

B31 (2017) 1750173 
Bibi, M., see Javed

Bing, P., Huang, S., Li, Z. Yu, Z., Lu, Y. \& Yao, J., Characteristic analysis of a photoexcited metamaterial perfect absorber at terahertz frequencies

Bisero, D., A first-principle model of the magnetic contrast in ultrathin films with perpendicular magnetization

Bogdanov, V.V., see Boiko

Boiko, Y.I., Bogdanov, V.V., Vovk, R.V., Kamchatnaya, S.N., Goulatis, I.L. \& Chroneos, A., Relaxation of the electric resistance in $\mathrm{YBa}_{2} \mathrm{Cu}_{3} \mathrm{O}_{7-x}$ single crystals at room temperature

Bordbar, G.H. \& Pouresmaeeli, F., Microscopic analysis of homogeneous electron gas by considering dipole-dipole interaction

Bordbar, G.H. \& Rastkhadiv, M.A., Variational calculations for ground state properties of liquid ${ }^{3} \mathrm{He}$ injected in a carbon nanotube

Botelho, L.C.L., A gaugeinvariant path integral for electrodynamics with magnetic monopoles in the Haddamard formalism

Botelho, L.C.L., A note on the electromagnetic irradiation in a holed spatial region: A space-time approach

Boughaleb, Y., see Benlattar

$\mathrm{Bu}, \mathrm{X}$., see Zhan

Cai, B., Guo, G. \& Lin, S., Multi-party quantum key agreement with teleportation

Cai, C., see Xue

Cai, G., see Zhang

Cai, K.-Q., Yu, L. \& Zhu, Y.B., A hybrid queuing strategy for network traffic on scale-free networks

Cai, R., Ye, J., Wang, Q., Chen, G., Liu, J., Shi, L.,
B31 (2017) 1750020

B31 (2017) 1750207

B31 (2017) 1750088

B31 (2017) 1750179

B31 (2017) 1750179

B31 (2017) 1750334

B31 (2017) 1750228

B31 (2017) 1750308

B31 (2017) 1750039

B31 (2017) 1750037

B31 (2017) 1750154

B31 (2017) 1750102

B31 (2017) 1750022

B31 (2017) 1750203

B31 (2017) 1750083
Lin, M., Xie, Z., Liang, J., Huang, H., Liu, J., Li, M., Liu, C. \& Song, H., Structural transition of carbon nanoparticles caused by energetic collisions

Cai, X., see Liang

Cai, X., see Zhang

Cambui, D.S., Collective behavior states in animal groups

Cao, C., see Chen

Cao, D., see Li

Cao, J., see Yang

Cao, L., see Chen

Cao, S., see $\mathrm{Hou}$

Carter, A.R., see Smith

Castle, R.S., see Feng

Castle, R.S., see Feng

Chai, B., see Yang

Chai, H.-P., Tian, B., Zhen, H.-L., Chai, J. \& Guan, Y.-Y., Analysis of the generalized $(2+1)$ dimensional NizhnikNovikov-Veselov equations with variable coefficients in an inhomogeneous medium

Chai, H.-P., see Wu

Chai, J., see Chai

Chai, J., see Yin

Chai, J., see Yuan

Chai, J., see Yu

Chai, J., see Zhao

Chan, A.H.S., see Rao

Chang, M., see Li

Chang, R., Application of multi-layer algorithm on image spam filtering

Chao, L.-M., Zhang, D. \& Pan, G., Roles of size and kinematics in drag reduction for two tandem flexible foils

Chary, M.N., see Sekhar

Chaturvedi, S.K., see Gargama

Chen, B., see Chu

Chen, B., see Zhang

Chen, C., see Zhang

Chen, D., Song, S., Zhang, D., Wang, P. \& Liu, W., Sensing characteristics of pure-shear film bulk acous-
B31 (2017) 1750347

B31 (2017) 1750241

B31 (2017) 1730004

B31 (2017) 1750054

B31 (2017) 1750139

B31 (2017) 1750219

B31 (2017) 1740040

B31 (2017) 1740016

B31 (2017) 1750251

B31 (2017) 1730001

B31 (2017) 1750282

B31 (2017) 1750029

B31 (2017) 1750349

B31 (2017) 1750135

B31 (2017) 1750122

B31 (2017) 1750135

B31 (2017) 1750132

B31 (2017) 1750100

B31 (2017) 1750258

B31 (2017) 1750013

B31 (2017) 1740019

B31 (2017) 1750327

B31 (2017) 1740030

B31 (2017) 1750311

B31 (2017) 1750180

B31 (2017) 1750301

B31 (2017) 1740085

B31 (2017) 1740081

B31 (2017) 1750220 
tic resonator in viscous liquids

Chen, F., Zhan, X., Gao, M., Tie, S. \& Gao, W. Anti-reflective microstructure array and its performance evaluation in thin film flexible solar cells

Chen, F., see Gao

Chen, F., see Zhan

Chen, G., Dou, X.-M. \& Zhu, X.-F., Extron prediction method based on improved period-3 feature strategy

Chen, G., Zhu, X., Chu, L., Cao, L. \& Lin, R., Detection method of the LGP warpage degree based on edge detection evaluation function

Chen, G., see Cai

Chen, G.-Q., see Zhu

Chen, J., Feng, B.-F. \& Chen, Y., Bilinear Bäcklund transformation, Lax pair and multi-soliton solution for a vector Ramani equation

Chen, J., Li, C., Huang, T. \& Yang, X., Global stabilization of memristorbased fractional-order neural networks with delay via output-feedback control

Chen, J., Li, P., Song, G. \& Ren, Z., Control of an innovative super-capacitorpowered shape-memoryalloy actuated accumulator for blowout preventer

Chen, J., see Chen

Chen, J., see Liu

Chen, J., see Zhan

Chen, L., Wang, Y., Huang, X., $\mathrm{Hu}, \mathrm{M} . \& \mathrm{Hu}, \mathrm{F}$., SA-SOM algorithm for detecting communities in complex networks

Chen, L., see Deng

Chen, L., see Gong

Chen, L., see Wang

Chen, L., see Wang

Chen, M. \& Li, B., Hybrid soliton solutions in the
B31 (2017) 1750086

B31 (2017) 1740001

B31 (2017) 1740002

B31 (2017) 1740003

B31 (2017) 1740079

B31 (2017) 1740016

B31 (2017) 1750347

B31 (2017) 1740062

B31 (2017) 1750133

B31 (2017) 1750031

B31 (2017) 1650426

B31 (2017) 1750158

B31 (2017) 1750146

B31 (2017) 1750154

B31 (2017) 1750262

B31 (2017) 1750079

B31 (2017) 1750286

B31 (2017) 1750178

B31 (2017) 1750193 $(2+1)$-dimensional nonlinear Schrödinger equation

Chen, M.N., see Tian

Chen, Q. \& Wang, Y., An infrared small target detection method based on nonnegative matrix factorization and compressed sensing

Chen, Q.-Y., Liu, M.Y., Huang, Y., Cao, C. \& He, Y., The atomic size effect on hybrid inorganic-organic perovskite $\mathrm{CH}_{3} \mathrm{NH}_{3} \mathrm{BI}_{3}(B=\mathrm{Pb}$, $\mathrm{Sn)}$ from first-principles study

Chen, S., see Hou

Chen, S., see Ma

Chen, S., see Qiu

Chen, S., see Wang

Chen, T., see Wang

Chen, T., see Zhu

Chen, W., see $\mathrm{Li}$

Chen, W., see Li

Chen, W., see Luo

Chen, W.-P., see Di

Chen, W.-P., see Fu

Chen, X., Li, Y., Tang, J., Wu, L., Liang, D. \& Zhang, R., First-principles study on electronic properties of stanene/ $\mathrm{WS}_{2}$ monolayer

Chen, X., see Ding

Chen, X., see Hou

Chen, X., see Liu

Chen, X., see Mao

Chen, X., see Qin

Chen, X., see Wen

Chen, X., see Zheng

Chen, X., see Zhu

Chen, Y., Luo, S., Zhang, M., Shen, H., Xin, F. \& Luo, Y., Modeling service time reliability in urban ferry system

Chen, Y., Yan, J., Yang, Y. \& Chen, J., Uncovering the community structure in signed social networks based on greedy optimization

Chen, Y., Zhao, Z., Liu, T., He, L., Di, Y. \& Cui, X.,
B31 (2017) 1750298

B31 (2017) 1750335

B31 (2017) 1740098

B31 (2017) 1750271

B31 (2017) 1750149

B31 (2017) 1750188

B31 (2017) 1750116

B31 (2017) 1750197

B31 (2017) 1750337

B31 (2017) 1750017

B31 (2017) 1740078

B31 (2017) 1740076

B31 (2017) 1750242

B31 (2017) 1750158 
Performance analysis on cascaded asymmetric photonic crystal Mach-Zehnder tunable filter

Chen, Y., Zou, L. \& Zhou, B., Diagonal rejection-based minimum variance distortionless response for fiber underwater acoustic array

Chen, Y., see Chen

Chen, Y., see Hou

Chen, Y., see Xu

Chen, Y., see Zhao

Chen, Z., Tian, W. \& Zhang, X., Analysis of a novel double driving signal line and driving electrodes separated RF MEMS switch

Chen, Z., see Liao

Chen, Z., see Liu

Chen, Z., see Liu

Chen, Z., see Zhang

Chen, Z.-C., see Li

Chen, Z.-Y., see Ding

Cheng, B., see Shen

Cheng, C., see Zhang

Cheng, C.-C., see Yang

Cheng, D., see Wang

Cheng, H., Qiu, C., Zhou, C., Sun, X. \& Yang, R., Experimental study of spreading characteristics of droplet impacting on canopy fabric surface

Cheng, H., see Qiu

Cheng, H., see Wang

Cheng, J.-T., see Wang

Cheng, Q.-B., see Zhao

Cheng, S., see Xie

Cheng, W., see Zhao

Cheng, X.-L., see Feng

Cheng, Y.W., see Si

Cheng, Y.Z., see Luo

Cheng, L. \& Zhang, Y., Lumptype solutions for the $(4+1)$-dimensional Fokas equation via symbolic computations

Choi, K.S., see Zhang

Chroneos, A., see Boiko

Chu, J., Rao, F. \& Gguo, J., Equivalent impedance method for determining the junction temperature of LED array
B31 (2017) 1750034

B31 (2017) 1740065
B31 (2017) 1750133
B31 (2017) 1750028
B31 (2017) 1750338
B31 (2017) 1750157

B31 (2017) 1750113

B31 (2017) 1750306

B31 (2017) 1750146

B31 (2017) 1750073

B31 (2017) 1750283

B31 (2017) 1750296

B31 (2017) 1750149

B31 (2017) 1740032

B31 (2017) 1750283

B31 (2017) 1750091

B31 (2017) 1750082

B31 (2017) 1750325

B31 (2017) 1750109

B31 (2017) 1750044

B31 (2017) 1750198

B31 (2017) 1750123

B31 (2017) 1750239

B31 (2017) 1740043

B31 (2017) 1750137

B31 (2017) 1750199

B31 (2017) 1750231

B31 (2017) 1750224

B31 (2017) 1850038

B31 (2017) 1750179

B31 (2017) 1740021
Chu, L., see Chen

Chu, L., see Wang

Chu, L., see Zhang

Chu, X.-J., see Wang

Chu, X.-J., see Wang

Chu, Z., Zhang, R., Chen, B. \& Li, H., Estimation of symmetrical components and their orthogonal components under unknown frequencies and unknown biases

Cong, L., Xu, L., Li, J., Wang, T. \& Han, Q., The conical conformal MEMS quasi-end-fire array antenna

Cui, C. \& Yang, D., Throughput optimization for dual collaborative spectrum sensing with dynamic scheduling

Cui, C.-M., see $\mathrm{Li}$

Cui, H., see Su

Cui, X., see Bian

Cui, X., see Chen

Cui, X.-J. \& Wang, L.-L., The process and mechanism of the GaN nanoparticles formed by nitridation of $\beta-\mathrm{Ga}_{2} \mathrm{O}_{3}$ crystal

Dahiya, S., see Sandeep

Dai, C.-J., see Qi

Dai, D., see Tian

Dai, H. \& Peng, J., The effects of welded joint characteristics on its properties in HDPE thermal fusion welding

Dai, L., Liu, C., Han, X., Wang, L., Tan, C., Yan, Z. \& Xu, Y., Dopant occupancy and UV-VIS-NIR spectroscopy of $\mathrm{Mg}(0,4,5$ and 6 mol.\%):Dy: $\mathrm{LiNbO}_{3}$ crystal

Dai, M., see Sun

Dai, X.H., see Song

Dang, M., see Zou

Daoud, M., see Achkir

Das, M.P., see Kim

Davarpanah, A.M., see Ghorbani

Demirhan, Y., see Nebioğlu

Deng, G.-F., see Jia
B31 (2017) 1740016

B31 (2017) 1750021

B31 (2017) 1750304

B31 (2017) 1750244

B31 (2017) 1750291

B31 (2017) 1740085

B31 (2017) 1750115

B31 (2017) 1740089

B31 (2017) 1740049

B31 (2017) 1750229

B31 (2017) 1750173

B31 (2017) 1750034

B31 (2017) 1750108

B31 (2017) 1750294

B31 (2017) 1750319

B31 (2017) 1750267

B31 (2017) 1750185

B31 (2017) 1750232

B31 (2017) 1750049

B31 (2017) 1750124

B31 (2017) 1750250

B31 (2017) 1750183

B31 (2017) 1750081

B31 (2017) 1750006

B31 (2017) 1750074

B31 (2017) 1750129 
Deng, G.-F., see Wang

Deng, G.-F., see Wang

Deng, H., Deng, J., Ma, M., Zhang, J., Yu, L. \& Wang, Z., 3D information detection with novel five composite fringe patterns

Deng, H., Wu, C. \& Wang, Y., A cognitive gateway based spectrum sharing method in downlink round robin scheduling of LTE system

Deng, J., see Deng

Deng, L., see Yan

Deng, Q., Li, Y., Shen, Y., Chen, L., Wang, G. \& Wang, S., Numerical simulation on $n-\mathrm{MoS}_{2} / p-\mathrm{Si}$ heterojunction solar cells

Deng, Y., see Fei

Deng, Y., see Zhang

Deng, Z., see Fang

Deng, Z., see Wang

Devenica, L.M., see Smith

Devi, E.C. \& Soibam, I., A correlated structural and electrical study of manganese ferrite nanoparticles with variation in sintering temperature

Di, X.-P., Chen, W.-P., Yin, L. \& Liu, X.-W., A 99.7-dB DR fourth-order sigmadelta modulator for digital gyroscope sensor

Di, X.-P., see Fu

Di, Y., see Chen

Di, Y., see Ye

Di, Y.-J., Shi, J.-P. \& Mao, G.-Y., A QR code identification technology in package auto-sorting system

Ding, L., Fan, W.-H., Chen, X., Chen, Z.-Y. \& Song, C., Terahertz spectroscopy and solid-state density functional theory calculations of structural isomers: Nicotinic acid, isonicotinic acid and 2-picolinic acid

Ding, L., Leung, V.C.M. \& Tan, M.-S., Robustness of complex networks with both unidirectional and bidirectional links against
B31 (2017) 1750216
B31 (2017) 1750012

B31 (2017) 1740088

B31 (2017) 1740070

B31 (2017) 1740088

B31 (2017) 1750273

B31 (2017) 1750079

B31 (2017) 1750243

B31 (2017) 1750195

B31 (2017) 1750249

B31 (2017) 1750021

B31 (2017) 1730001

B31 (2017) 1750236

B31 (2017) 1750097

B31 (2017) 1750064

B31 (2017) 1750034

B31 (2017) 1750333

B31 (2017) 1740035

B31 (2017) 1750149 cascading failures

Ding, P., see Wang

Ding, X., see Wang

Ding, Y., see Liang

Dixit, A. \& Pandey, P.C., Off-axis photonic bands of hexagonal plasma photonic crystal fiber containing elliptical holes with defect of high index material for nonlinear waves by PWE method

Dong, G., see Wang

Dong, G., see Zhang

Dong, H., see $\mathrm{Su}$

Dong, H., see Yang

Dong, H.-Y., see Li

Dong, J.-J. \& Li, P., The $a$-cycle problem in $\mathrm{XY}$ model with ring frustration

Dong, L. \& Hu, Y., A lowvoltage, low-power voltage reference based on subthreshold MOSFETS

Dong, L. \& Hu, Y., Microfluidic networks embedded in a printed circuit board

Dong, L., see Jiang

Dong, L., see Liu

Dong, M.-J., Tian, S.-F., Yan, X.-W., Zou, L. \& Li, J., Solitary waves, rogue waves and homoclinic breather waves for a $(2+1)$-dimensional generalized KadomtsevPetviashvili equation

Dong, M.-J., see Yan

Dong, X., see Xing

Dong, Y., Yao, H., Du, J., Zhao, J. \& Jiang, J., Research on local resonance and Bragg scattering coexistence in phononic crystal

Dong, Y.-C., see Zhu

Dong, Y.Z., see Duan

Dong, Z., Fang, Y. \& Tian, M., Cascading failures of interdependent networks with different $k$-core structures

Dong, Z., see Tian

Dong, Z.-W., see Li

Dou, X.-M., see Chen
B31 (2017) 1750252

B31 (2017) 1750202

B31 (2017) 1750021

B31 (2017) 1750241

B31 (2017) 1750156

B31 (2017) 1750354

B31 (2017) 1750222

B31 (2017) 1650428

B31 (2017) 1750230

B31 (2017) 1750310

B31 (2017) 1750061

B31 (2017) 1740069

B31 (2017) 1740017

B31 (2017) 1740004

B31 (2017) 1740011

B31 (2017) 1750281

B31 (2017) 1750350

B31 (2017) 1750212

B31 (2017) 1750127

B31 (2017) 1740027

B31 (2017) 1750144

B31 (2017) 1750112

B31 (2017) 1750267

B31 (2017) 1750077

B31 (2017) 1740079 
Dowran, M., see Gh. Khorrami

Drize, A. \& Settaouti, A., Three-dimensional Monte Carlo simulations of materials on the physical deposition process

Du, A., see Gao

Du, J., see Dong

Du, J.-M., see Zhu

Du, M.-M., see Wang

Du, X., see Gao

Du, X., see Li

Du, Z., see Yang

Du, Z., see Yang

$\mathrm{Du}, \mathrm{Z}$., see $\mathrm{Yu}$

Duan, H., Lin, Z.J., Dong, Y.Z. \& Huang, Y., Evaluation of the role of oxygen vacancies in $\mathrm{La}_{3} \mathrm{GaGe}_{5}$ $\mathrm{O}_{1} 6: \mathrm{Tb}^{3+}$ persistent phosphor

Duan, J.Z., see Ling

Duan, R., see Wang

Duan, W.S., see Ling

Ebrahim, S., see Anas

Eisa, M.H., see Ibraheem

Ejderha, K., see Kocyigit

Eldeen, I.G., see Anas

Elkoraychy, E., see Benlattar

En, Y.-F., see Liu

En, Y.-F., see Liu

Eshghi, H., see Heidaryan

Fan, C., see Wang

Fan, H., see Liu

Fan, H.-Y., see Wu

Fan, P., see Zhou

Fan, W.-H., see Ding

Fan, X., see Wei

Fan, X., see Zhang

Fan, Y., see Yong

Fan, Y., see Zhang

Fang, C., Mu, D., Deng, Z. \& Yan, J., Uncovering the fuzzy community structure accurately based on steepest descent projection

Fang, C.-H., see Wang

Fang, H., Zhang, F.-P., Ruan, X.-X., Huang, C.-S., Jiang, Z.-N., Peng, J.-Y. \& Wang, R.-Z., Strain-induced negative differential resistance in ultrasmall carbon nanotube
B31 (2017) 1750175


B31 (2017) 1750165
B31 (2017) 1750016
B31 (2017) 1750127
B31 (2017) 1740062
B31 (2017) 1750336
B31 (2017) 1750093
B31 (2017) 1750085
B31 (2017) 1750201
B31 (2017) 1750349
B31 (2017) 1750258

B31 (2017) 1750144

B31 (2017) 1750248

B31 (2017) 1750044

B31 (2017) 1750248

B31 (2017) 1750290

B31 (2017) 1750068

B31 (2017) 1750288

B31 (2017) 1750290

B31 (2017) 1750037

B31 (2017) 1740007

B31 (2017) 1740020

B31 (2017) 1750227

B31 (2017) 1750202

B31 (2017) 1740041

B31 (2017) 1750151

B31 (2017) 1750184

B31 (2017) 1750149

B31 (2017) 1750166

B31 (2017) 1740042

B31 (2017) 1750276

B31 (2017) 1750147

B31 (2017) 1750249

B31 (2017) 1750244

B31 (2017) 1750217
Fang, W.-X., see Liu

Fang, X.Y., see Song

Fang, Y., see Dong

Fang, Z.-J., Zhai, X.-S., Li, Z.-L., Pan, R.-J. \& Mo, M., Pressure dependence of the electronic structure in kaolinite: A firstprinciples study

Faraji, E., Baghshahi, H.R. \& Tavassoly, M.K., The influence of atomic dipoledipole interaction on the dynamics of the population inversion and entanglement of two atoms interacting non-resonantly with two coupled modes field

Farzi, G., see Ghamari

Fei, L., Mo, H. \& Deng, Y., A new method to identify influential nodes based on combining of existing centrality measures

Fei, X., see Xi

Feng, B.-F., see Chen

Feng, H., see Li

Feng, L., Castle, R.S. \& Li, Y., XUV pulse effect on harmonic emission spectra and attosecond pulse generation

Feng, L., Li, Y., Meng, F., Liu, H. \& Castle, R.S., High-order harmonic and attosecond pulse generations from Rydberg state driven by the spatially inhomogeneous field

Feng, L.-L., see Zou

Feng, M., see Lv

Feng, R., see Jie

Feng, S.-Q., Yang, Y., Li, J.Y., Jiang, X.-X., Li, H.-N. \& Cheng, X.-L., Pressure effect on the hardness of diamond and $\mathrm{W}_{2} \mathrm{~B}_{5}$ : firstprinciple calculations

Feng, X., see Zeng

Feng, Z., see $\mathrm{Hu}$

Fu, F., see Yin

$\mathrm{Fu}, \mathrm{H}$., see Wen

Fu, Q., Di, X.-P., Chen, W.P., Yin, L. \& Liu, X.-W.,
B31 (2017) 1740020

B31 (2017) 1750124

B31 (2017) 1750112

B31 (2017) 1750194

B31 (2017) 1750038 B31 (2017) 1750120

B31 (2017) 1750243

B31 (2017) 1740094

B31 (2017) 1750133

B31 (2017) 1750090

B31 (2017) 1750282

B31 (2017) 1750029

B31 (2017) 1750348

B31 (2017) 1740091

B31 (2017) 1740023

B31 (2017) 1750137

B31 (2017) 1750238

B31 (2017) 1750182

B31 (2017) 1750150

B31 (2017) 1750056 
A temperature characteristic research and compensation design for micromachined gyroscope

$\mathrm{Fu}, \mathrm{X}$., see Qin

$\mathrm{Fu}, \mathrm{X}$., see Zou

$\mathrm{Fu}, \mathrm{Y}$., see $\mathrm{Li}$

$\mathrm{Fu}, \mathrm{Y}$., see $\mathrm{Li}$

Ga, Y.-T., see Wang

Gao, F., Du, X., Wu, F., Li, X., Hu, X. \& Song, H., Thermoelectric properties of $\mathrm{Cu}_{2} \mathrm{Se} / x \mathrm{Ni}_{0.85} \mathrm{Se}$ hotpressed from hydrothermal synthesis nanopowders

Gao, G., Comment on "Cryptanalysis and improvement of multiparty semiquantum secret sharing based on rearranging orders of qubits"

Gao, H., see Teng

Gao, L., see Wang

Gao, M., Zhan, X., Chen, F., Si, Y., Tie, S. \& Gao, W., Fabricating omnidirectional low-reflection films by nanoimprinting method for boosting solar power generation of silicon-based solar cells

Gao, M., see Chen

Gao, M., see Zhan

Gao, N. \& Hou, H., Low frequency acoustic properties of a honeycombsilicone rubber acoustic metamaterial

Gao, N., Hou, H. \& Xin, H., A single and double slotting radial acoustic metamaterial plate

Gao, P.-F., see Wen

Gao, Q.-H., Du, A. \& Yang, Z.-J., Structural inheritance and difference between $\mathrm{Ti}_{2} \mathrm{AlC}, \mathrm{Ti}_{3} \mathrm{AlC}_{2}$ and $\mathrm{Ti}_{5} \mathrm{Al}_{2} \mathrm{C}_{3}$ under pressure from first principles

Gao, T., see Guo

Gao, W., see Chen

Gao, W., see Gao

Gao, W., see Zhan

Gao, X.-Y., see Jiang

Gao, Y., see Sun

\section{B31 (2017) 1750064 \\ B31 (2017) 1750337 \\ B31 (2017) 1750250 \\ B31 (2017) 1750140 \\ B31 (2017) 1750270 \\ B31 (2017) 1750216}

B31 (2017) 1750093

B31 (2017) 1775001

B31 (2017) 1750204

B31 (2017) 1750044

B31 (2017) 1740002

B31 (2017) 1740001

B31 (2017) 1740003

B31 (2017) 1750118

B31 (2017) 1750128

B31 (2017) 1750017

B31 (2017) 1750016

B31 (2017) 1750245

B31 (2017) 1740001

B31 (2017) 1740002

B31 (2017) 1740003

B31 (2017) 1750254

B31 (2017) 1750080
Gao, Y.-T., see Huang

Gao, Y.-T., see $\mathrm{Hu}$

Gao, Y.-T., see Jiang

Gao, Y.-T., see Jia

Gao, Y.-T., see Wang

Gao, Z., Luan, B., Zhao, J. \& Liu, X., An integrated low $1 / f$ noise and high-sensitivity CMOS instrumentation amplifier for TMR sensors

Gao, Z., see Li

Gao, Z., see Wang

Gao, Z.-Y., see Wang

Gao, Z.-Y., see Wang

Gao, Z.-Y., see Wang

Gargama, H., Thakur, A.K. \& Chaturvedi, S.K., Microwave characterization of nickel-based nanocomposites - high EMI shielding and radar absorption capability

Garibli, A., see Huseynov

Ge, D.Y., see Song

Geng, X., see Liu

Geng, X.-M., Mi, S.-C., Wang, T.-J., He, L.-Y. \& Wang, C., Plasmonic bandpass filter device using coupled asymmetric crossshaped cavity

Gersappe, D., see Sun

Gguo, J., see Chu

Gh. Khorrami, H., Mousavi, M. \& Dowran, M., Structural and optical properties of KNN nanoparticles synthesized by a sol-gel combustion method

Gh. Solookinejad Jabbari, M., Sangachin, E.A. \& Asadpour, S.H., Absorption and dispersion management of near-infrared probe light in the carbon nanotube quantum dot nanostructure with spinorbit coupling

Ghamari, M. \& Farzi, G., Frequency and composition dependency of optical and dielectric properties of PMMA/boehmite nano-hybrid prepared via
B31 (2017) 1750126
B31 (2017) 1750035
B31 (2017) 1750254
B31 (2017) 1750129
B31 (2017) 1750012

B31 (2017) 1750070

B31 (2017) 1750030

B31 (2017) 1750082

B31 (2017) 1750104

B31 (2017) 1750244

B31 (2017) 1750291

B31 (2017) 1750301

B31 (2017) 1750257

B31 (2017) 1750124

B31 (2017) 1750299

B31 (2017) 1750001

B31 (2017) 1650431

B31 (2017) 1740021

B31 (2017) 1750175

B31 (2017) 1750322 
facile aqueous one-pot process

Ghorbani, M.H. \& Davarpanah, A.M., Synthesis and characterization of $\mathrm{MnO}_{2}$ nanowires

Gong, B., Guo, F., Zou, W., Chen, L., Song, K. \& Zhao, $X$., New design of multiband negative-index metamaterial and absorber at visible frequencies

Gong, L.J., Pan, C.L. \& Pan, Q.S., Analysis of the actuating effects of triplelayer piezoelectric cantilevers considering electromechanical coupling correction

Gong, Y., see Liu

Goulatis, I.L., see Boiko

Gu, D., see $\mathrm{Li}$

Gu, L., see Zhang

Gu, M., see Zheng

Gu, W., Wen, Z. \& Xu, Y., Low voltage electrophoresis chip with multisegments synchronized scanning

Gu, X., see Yao

$\mathrm{Gu}, \mathrm{Z}$., see Peng

Guan, D., see Hou

Guan, H., see Zhu

Guan, J., see Xing

Guan, L., see Wang

Guan, Y.-Y., see Chai

Guo, F., see Gong

Guo, G., see Cai

Guo, J. \& Zhang, R., Immunomagnetic separation for MEMS-based biosensor of waterborne pathogens detection

Guo, J., see Yang

Guo, J., see Zhao

Guo, J.X., see Song

Guo, L., see Liu

Guo, L.-F. \& Li, P., Topological phases characterized by spin Chern number and skyrmion number in triangular BoseHubbard model

Guo, P., see Luo

Guo, X., see Yuan
B31 (2017) 1750221

B31 (2017) 1750120

B31 (2017) 1750006

B31 (2017) 1750286

B31 (2017) 1750024
B31 (2017) 1750266
B31 (2017) 1750179
B31 (2017) 1750085
B31 (2017) 1750147
B31 (2017) 1740044

B31 (2017) 1750048

B31 (2017) 1740025

B31 (2017) 1750103

B31 (2017) 1750251

B31 (2017) 1740048

B31 (2017) 1750212

B31 (2017) 1750021

B31 (2017) 1750135

B31 (2017) 1750286

B31 (2017) 1750102

B31 (2017) 1740084

B31 (2017) 1740024

B31 (2017) 1740043

B31 (2017) 1750124

B31 (2017) 1750116

B31 (2017) 1750341

B31 (2017) 1750045
Guo, Y., Gao, T. \& Li, S., The structural, electronic, mechanical, lattice dynamics and thermodynamic properties of $\mathrm{Rh}_{5} \mathrm{~B}_{4}$ : firstprinciples calculations

Guo, Y., see Liu

Guo, Y.-F., Shen, Y.-J., Xi, B. \& Tan, J.-G., Colored correlated multiplicative and additive Gaussian colored noises-induced transition of a piecewise nonlinear bistable model

Guo, Y.-J., see Zhao

Guo, Z.-G., see Jin

Güllü, H.H. \& Parlak, M., Structural characterization of $\mathrm{Zn}-\mathrm{In}-\mathrm{Se}$ thin films

Hameed, A., see Sekhar

Hamidnia, M., Luo, Y., Wang, X. \& Li, C., Experimental investigation on the thermal performance of Si micro-heat pipe with different cross-sections

Han, B., see Zhao

Han, L., see Liang

Han, Q., see Cong

Han, S., see Yang

Han, W., see Shi

Han, X., see Dai

Han, X., see Liu

Han, X., see Zhao

Han, Y., Zhai, M. \& Zhou, J., A thermal protection module for automotive integrated circuits

Han, Y., see Liang

Hao, H.-Q., see Zhao

Hao, L., see Pang

Hao, Y., see Wang

Hao, Y., see Zhang

Hassan, Z., see Mahyuddin

Hayat, S.S., Rehman, Z. \& Shah, Z.A., A study of dynamical evolution of small two-dimensional copper islands' diffusion on $\operatorname{Ag}\left(\begin{array}{lll}1 & 1 & 1\end{array}\right)$ surface and observed surface effects

He, D. \& Liu, S., Effect of heat treatment on structure, surface composition, infrared emission and sur-
B31 (2017) 1750279

B31 (2017) 1750245

B31 (2017) 1750073

B31 (2017) 1750256

B31 (2017) 1750013

B31 (2017) 1740095

B31 (2017) 1750043

B31 (2017) 1750180

B31 (2017) 1750157

B31 (2017) 1750241

B31 (2017) 1750115

B31 (2017) 1750230

B31 (2017) 1740096

B31 (2017) 1750232

B31 (2017) 1740018

B31 (2017) 1750312

B31 (2017) 1740097

B31 (2017) 1750241

B31 (2017) 1750174

B31 (2017) 1750101

B31 (2017) 1750354

B31 (2017) 1750222

B31 (2017) 1750313

B31 (2017) 1750316 
face electrical properties of tourmaline

He, G., see Liu

He, H., see Peng

He, J. \& Zhang, Q., Study of averaged collision strength of non-Maxwellian distribution in plasma

He, J., see Zhao

$\mathrm{He}$, J., see Zhen

$\mathrm{He}$, L., see Chen

He, L.-Y., see Geng

$\mathrm{He}$, S., see Zhang

He, X., see Jiang

$\mathrm{He}, \mathrm{X}$., see $\mathrm{Li}$

He, Y. \& Ma, W., Two robust quantum key agreement protocols based on logical GHZ states

He, Y., Zhang, B. \& Shen, J., Performance of terahertz metamaterials as highsensitivity sensor

$\mathrm{He}$, Y., see Chen

$\mathrm{He}$, Y., see Li

He, Z., Bai, J. \& Ma, C., Conductance through a parallel-coupled double quantum dot with a sidecoupled quantum dot system

He, Z., see Xue

He, Z.-C., see Zhang

Heidaryan, N. \& Eshghi, H., An investigation on physical properties of $\mathrm{SiO}_{x}$ nanowires deposited by chemical vapor deposition method: The effect of substrate to boat distance

Hoch, M.J.R., Spin clusters and low-energy excitations in rare earth Kagome systems

Hong, N.V., see Yen

Hou, H., see Gao

Hou, H., see Gao

Hou, J., see Liu

Hou, J.-X., Zhang, Y.-H., Chen, Y. \& Yu, X.-C., Translocation of polymer chain in post array induced by arrangement differ

Hou, M., Wu, J.H., Cao, S., Guan, D. \& Zhu, Y.,
B31 (2017) 1750026
B31 (2017) 1750300
B31 (2017) 1750103

B31 (2017) 1750213

B31 (2017) 1750123

B31 (2017) 1750292

B31 (2017) 1750034

B31 (2017) 1750001

B31 (2017) 1750087

B31 (2017) 1740033

B31 (2017) 1750090

B31 (2017) 1750015

B31 (2017) 1750240

B31 (2017) 1750139

B31 (2017) 1750085

B31 (2017) 1750095

B31 (2017) 1750022

B31 (2017) 1740081

B31 (2017) 1750227

B31 (2017) 1630010

B31 (2017) 1750036

B31 (2017) 1750118

B31 (2017) 1750128

B31 (2017) 1750352

B31 (2017) 1750028
Extremely low frequency band gaps of beam-like inertial amplification metamaterials

Hou, Q.-Y., see Sun

Hou, S., Xiong, Y., Chen, S., Chen, X., Xiong, P., Tian, Y. \& Liu, G., Effect of current density on the deposit stress in gold electroplating

Hou, Z., see Li

Hou, Z.-Y., see Shi

Hu, F., see Chen

Hu, G.C., see Tian

$\mathrm{Hu}, \mathrm{K} .-$ Y., see Zhang

$\mathrm{Hu}$, M., see Chen

Hu, M.-B., see Zhang

Hu, P., Zhang, W., Hu, Z., Feng, Z., Ma, L., Zhang, X., Sheng, X., Luo, L. \& Wang, Y., Luminescence and cathodoluminescent properties of monoclinic $\mathrm{Y}_{2} \mathrm{WO}_{6}$ co-doped with Dy-Bi

Hu, S.-L., see Li

$\mathrm{Hu}, \mathrm{W}$., see $\mathrm{Si}$

Hu, W.-Q., Gao, Y.-T., Zhao, C. \& Lan, Z.-Z., Breathers and rogue waves for an eighth-order nonlinear Schrödinger equation in an optical fiber

Hu, W.-Q., see Jia

Hu, W.-Q., see Wang

$\mathrm{Hu}, \mathrm{X}$., see Gao

$\mathrm{Hu}, \mathrm{X}$., see Liu

$\mathrm{Hu}, \mathrm{X}$., see Wang

$\mathrm{Hu}, \mathrm{X}$., see Zhang

Hu, Y., Xiong, C. \& Li, B., A $0.975 \mu \mathrm{w}$ 10-bit $100 \mathrm{kS} / \mathrm{s}$ SAR ADC with an energyefficient and area-efficient switching scheme

$\mathrm{Hu}$, Y., see Dong

$\mathrm{Hu}, \mathrm{Y}$, see Dong

$\mathrm{Hu}$, Y., see Zou

Hu, Y.-H., see Jin

Hu, Y.-L., see Zhai

$\mathrm{Hu}, \mathrm{Z}$., see $\mathrm{Hu}$

Hua, H.L., see Tian

Huang, C., see Wang

Huang, C.-S., see Fang

Huang, G.-F., see Si
B31 (2017) 1750251

B31 (2017) 1750200

B31 (2017) 1750188

B31 (2017) 1750004

B31 (2017) 1750247

B31 (2017) 1750262

B31 (2017) 1750335

B31 (2017) 1750117

B31 (2017) 1750262

B31 (2017) 1750063

B31 (2017) 1750182

B31 (2017) 1750310

B31 (2017) 1750199

B31 (2017) 1750035

B31 (2017) 1750129

B31 (2017) 1750216

B31 (2017) 1750093

B31 (2017) 1750266

B31 (2017) 1750309

B31 (2017) 1750051

B31 (2017) 1740051

B31 (2017) 1740017

B31 (2017) 1740069

B31 (2017) 1750293

B31 (2017) 1740095

B31 (2017) 1740050

B31 (2017) 1750182

B31 (2017) 1750335

B31 (2017) 1750143

B31 (2017) 1750217

B31 (2017) 1650429 
Huang, H., see Cai

Huang, H.-J., see Xiao

Huang, H.-J., see Xiao

Huang, J., see Liu

Huang, L., see Zhao

Huang, Q.-M. \& Gao, Y.-

T., Bilinear form, bilin-

ear Bäcklund transforma-

tion and dynamic features

of the soliton solutions

for a variable-coefficient

$(3+1)$-dimensional gener-

alized shallow water wave equation

Huang, S., Wang, Y. \& Ji, Z., Multi-objective particle swarm optimization using Pareto-based set and aggregation approach

Huang, S., see Bing

Huang, S., see Wang

Huang, T., see Chen

Huang, W., Algorithm for determination of sample size using Linex loss function

Huang, W.-Q., see Si

Huang, X., see Chen

Huang, Y., Li, W., Wang, G. \& Yong, X., Lie symmetry analysis of the deformed $\mathrm{KdV}$ equation

Huang, Y., see Chen

Huang, Y., see Duan

Huang, Y., see Xu

Huang, Y., see Yong

Huang, Y., see Zhu

Huang, Z.X., see Ma

Huo, Y., see Pang

Huseynov, E. \& Garibli, A., The neutron irradiation effects on the temperature dependencies of the electrical conductivity of nanosilicon particles

Huseynov, E., see Samadov

Ibraheem, A.M., Eisa, M.H., Adlan, W., Amolo, G.O. \& Khalafalla, M.A.H., Firstprinciples identification of charge-transition levels of native defects in $\mathrm{BaF}_{2}$

Ibrahim, A.M., see AbdelRahman

Inc, M., Aliyu, A.I. \& Yusuf, A., Dark optical, singu-

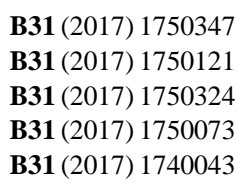

B31 (2017) 1750126

B31 (2017) 1740073

B31 (2017) 1750207

B31 (2017) 1740099

B31 (2017) 1750031

B31 (2017) 1740060

B31 (2017) 1650429

B31 (2017) 1750262

B31 (2017) 1750275

B31 (2017) 1750139

B31 (2017) 1750144

B31 (2017) 1750338

B31 (2017) 1750276

B31 (2017) 1740076

B31 (2017) 1750018

B31 (2017) 1750101

B31 (2017) 1750257

B31 (2017) 1750134

B31 (2017) 1750068

B31 (2017) 1750255 lar solitons and conservation laws to the nonlinear Schrödinger's equation with spatio-temporal dispersion

Ipekoğlu, U., see Yurtseven

Ismayilova, N.A., Orudzhev, G.S. \& Jabarov, S.H., Firstprinciple calculation of the electronic structure, DOS and effective mass $\mathrm{TlInSe}_{2}$

Jabarov, S.H., see Ismayilova

Jabbari, M., see Gh. Solookinejad

Javed, Q., Baqi, S., Abbas, H. \& Bibi, M., A facile growth mechanism, structural, optical, dielectric and electrical properties of $\mathrm{ZnSe}$ nanosphere via hydrothermal process

Jeong, Y., see ur Rehman

Ji, L., see Liu

Ji, R., see Zhu

Ji, Z., see Huang

Ji, Z., see Wang

Ji, Z., see Wang

Ji, Z., see Wang

Ji, Z., see Wang

Ji, Z., see $\mathrm{Xu}$

$\mathrm{Ji}, \mathrm{Z}$., see $\mathrm{Xu}$

Ji, Z., see Zhang

Jia, B., see Wang

Jia, B., see Wang

Jia, B., see Wang

Jia, B., see Wang

Jia, B., see Zhang

Jia, G., see Liang

Jia, L., see Liu

Jia, L., see Su

Jia, L., see Yang

Jia, S.-L., Gao, Y.-T., Hu, W.Q., Su, J.-J. \& Deng, G.F., Solitons and breather waves for a $(2+1)$-dimensional Sawada-Kotera equation

Jia, S.-L., see Wang

Jia, S.-L., see Wang

Jia, X., see Jiang

Jia, X., see Ma

Jia, Y., see Luo

Jia, Z., see Wen

Jia, Z.W., Jiang, T. \& Liu, Y.,

Three-phase receiving coil
B31 (2017) 1750163 B31 (2017) 1750092

B31 (2017) 1750155 B31 (2017) 1750155

B31 (2017) 1750322

B31 (2017) 1750020

B31 (2017) 1750119

B31 (2017) 1750300

B31 (2017) 1740008

B31 (2017) 1740073

B31 (2017) 1740072

B31 (2017) 1740071

B31 (2017) 1740099

B31 (2017) 1740057

B31 (2017) 1740058

B31 (2017) 1740100

B31 (2017) 1740087

B31 (2017) 1750104

B31 (2017) 1750244

B31 (2017) 1750291

B31 (2017) 1750353

B31 (2017) 1750063

B31 (2017) 1750241

B31 (2017) 1750339

B31 (2017) 1650428

B31 (2017) 1750230

B31 (2017) 1750129

B31 (2017) 1750216

B31 (2017) 1750012

B31 (2017) 1750261

B31 (2017) 1750018

B31 (2017) 1750341

B31 (2017) 1750056 
of wireless power transmission system for gastrointestinal robot

Jiang, C. \& He, X., An improved PolSAR image speckle reduction algorithm based on LMMSE and RICA

Jiang, J., see Dong

Jiang, L.-X., see Li

Jiang, Q., see Qiu

Jiang, R., see Zhang

Jiang, T., see Jia

Jiang, W., see Zhang

Jiang, X., see Wu

Jiang, X., see Zhu

Jiang, X.-H., Gao, Y.-T. \& Gao, X.-Y., Investigation on the behaviors of the soliton solutions for a variable-coefficient generalized $A B$ system in the geophysical flows

Jiang, X.-X., see Feng

Jiang, Y., Bi, H., Dong, L. \& Li, Q., Reliability challenge of ESD protection: from planner SOI MOSFET to SOI FinFET

Jiang, Y., Jia, X. \& Ma, H., The thermoelectric properties of $\mathrm{CoSb}_{3}$ compound doped with Te and Sn synthesized at different pressure

Jiang, Y.-R., see Li

Jiang, Z.-N., see Fang

Jiao, Z., see Sun

Jie, G., Ma, J. \& Feng, R., A non-contact method for determining the junction temperature of GaN-based blue light LED

Jin, L., Zhang, G. \& Zhu, X., Formal analysis and evaluation of the back-off procedure in IEEE802.11P VANET

Jin, X.-G., Shou, G.-C., Hu, Y.-H. \& Guo, Z.-G., Service entity network virtualization architecture and model

Jin, Y., see Xu

John, R., see Joseph

\section{B31 (2017) 1750277}

B31 (2017) 1740033

B31 (2017) 1750127

B31 (2017) 1750296

B31 (2017) 1750192

B31 (2017) 1750063

B31 (2017) 1750277

B31 (2017) 1750220

B31 (2017) 1740046

B31 (2017) 1740037

B31 (2017) 1750254

B31 (2017) 1750137

B31 (2017) 1740004

B31 (2017) 1750261

B31 (2017) 1750296

B31 (2017) 1750217

B31 (2017) 1750295

B31 (2017) 1740023

B31 (2017) 1740063

B31 (2017) 1740095

B31 (2017) 1750075

B31 (2017) 1750287
Joseph, S., Kumar, A.V.R. \& John, R., Non-aqueous electrochemical deposition of lead zirconate titanate films for flexible sensor applications

Joshi, J.H., see Kochuparampil

Joshi, M.J., see Kochuparampil

Ju, W., see Su

Jun, S., see Zhou

Jun, W., see Zhou

Kadhim, I.H. \& Abu Hassan, H., Effect of aging heat time and annealing temperature on the properties of nanocrystalline tin dioxide thin films

Kamchatnaya, S.N., see Boiko

Kang, L., see Zhang

Kantar, E., Dynamic calculations of the core/shell structured Ising-type endohedral fullerenes: the effect of core and core/shell interaction

Karaaslan, M., see Akgol

Karaaslan, M., see Altintas

Karadag, F., see Altintas

Karaduman, I. \& Acar, S., The gas sensing properties of hafnium oxide thin films depending on the annealing environment

Karimipour, M., Bagheri, M. \& Molaei, M., Enhancement of durability of NIR emission of $\mathrm{Ag}_{2} \mathrm{~S} @ \mathrm{ZnS}$ QDs in water

Kazerani, M.R., see Mardani

Khajavi, R., see Kordani

Khalafalla, M.A.H., see Ibraheem

Kim, S.-H., Sy, P.-V. \& Das, M.P., Acoustic Eaton lens array and its fluid application

Kislov, A., Vibrational dynamics of alpha-quartz with neutral silicon vacancies

Kochuparampil, A.P., Joshi, J.H. \& Joshi, M.J., Growth,
B31 (2017) 1750287

B31 (2017) 1750246

B31 (2017) 1750246

B31 (2017) 1750229

B31 (2017) 1740082

B31 (2017) 1740082

B31 (2017) 1750114

B31 (2017) 1750179

B31 (2017) 1730004

B31 (2017) 1750307

B31 (2017) 1750176

B31 (2017) 1750274

B31 (2017) 1750274

B31 (2017) 1750284

B31 (2017) 1750297

B31 (2017) 1750094

B31 (2017) 1750235

B31 (2017) 1750068

B31 (2017) 1750081

B31 (2017) 1750315 
structural, spectroscopic, thermal, dielectric and optical study of cobalt sulphide-doped ADP crystals

Kocyigit, A., Ozturk, E., Ejderha, K. \& Turgut, G., Effect of different sound atmospheres on $\mathrm{SnO}_{2}: \mathrm{Sb}$ thin films prepared by dip coating technique

Kolesnikov, S.V., see Sidorenkov

Kolesnikov, S.V., see Tsysar

Konobeeva, N. \& Belonenko, M., Conductivity of impurity graphene nanoribbons and gate electric field

Konobeeva, N.N. \& Belonenko, M.B., Threedimensional few-cycle optical Airy pulses in the array of carbon nanotubes with multilevel impurities

Kordani, N., Alizadeh, M., Lohrasby, F., Khajavi, R., Baharvandi, H.R., Rezanejad, M. \& Ahmadzadeh, M., Mechanical properties of composites made of hybrid fabric impregnated with silica nanoparticles and epoxy resin

Kou, S.-P., see Zhao

Kou, Y., Sang, A., Li, X. \& Wang, X., Fabrication of self-enclosed nanochannels based on capillary-pressure balance mechanism

Kuai, J., Ardashev, D.V. \& Zhang, H., Study of $\alpha$ $\mathrm{Fe}_{2} \mathrm{O}_{3}$ formation and its measurement in oxide films of wheel surface during ELID grinding process

Kumar, A.V.R., see Joseph

Kumar, M., see Bhatt

Kumar, R., see Bhatt

Kunze, H.J., see Akel

Kurths, J., see Zhao

Lai, P.-T., see Liu

Lakhno, V., Peculiarities in the concentration dependence of the superconduct- ing transition temperature in the bipolaron theory of

B31 (2017) 1750246

B31 (2017) 1750288

B31 (2017) 1750289

B31 (2017) 1750142

B31 (2017) 1750340

B31 (2017) 1750005

B31 (2017) 1750235

B31 (2017) 1750123

B31 (2017) 1750253

B31 (2017) 1750287

B31 (2017) 1750011

B31 (2017) 1750011

B31 (2017) 1750167

B31 (2017) 1750084

B31 (2017) 1750332 Cooper pairs

Lan, M.T., see Yen

Lan, Z.-Z., see $\mathrm{Hu}$

Lan, Z.-Z., see Wang

Lang, G., Ma, L. \& Xu, Y., Performance analysis of IEEE 802.11n network under unsaturated conditions

Lang, J., see Zhang

Lee, S., see Akel

Lei, M., see Wang

Leng, H., Ao, P., Zhu, J.-R. \& Tang, H.-G., Combination forecasting method and application based on the fractal dimension weight

Leung, V.C.M., see Ding

$\mathrm{Li}, \mathrm{A}$., see $\mathrm{Li}$

Li, A., see Zhao

Li, A.-L., Zhang, C., Wang, H., He, Y., Sun, D., Wang, L., Du, X. \& Gu, D., Design of temperatureimmunization system packaging for the resonant pressure sensor

Li, B. \& Li, X., Study on degenerate coefficient and degeneration evaluation of lithium-ion battery

Li, B., see Chen

Li, B., see $\mathrm{Hu}$

Li, B., see Liu

Li, C., see Chen

Li, C., see Hamidnia

Li, C., see Song

Li, C., see Sun

Li, C., see Yang

Li, D., Fu, Y. \& Yang, L., Coupling dynamic modeling and simulation of three-degree-of-freedom micromanipulator based on piezoelectric ceramic of fuzzy PID

B31 (2017) 1750125

B31 (2017) 1750036

B31 (2017) 1750035

B31 (2017) 1750012

B31 (2017) 1740093

B31 (2017) 1750220

B31 (2017) 1750167

B31 (2017) 1750354

B31 (2017) 1740053

B31 (2017) 1750252

B31 (2017) 1750310

B31 (2017) 1750084

B31 (2017) 1750085

B31 (2017) 1740092

B31 (2017) 1750298

B31 (2017) 1740051

B31 (2017) 1740007

B31 (2017) 1750031

B31 (2017) 1750279

B31 (2017) 1750280

B31 (2017) 1750200

B31 (2017) 1750189

Li, D., Wang, S. \& Fu, Y., Quality detection system and method of microaccessory based on microscopic vision

Li, D., see Shang

Li, D., see Xue
B31 (2017) 1750270

B31 (2017) 1750059

B31 (2017) 1750022
B31 (2017) 1750140 
Li, H. \& Shen, Y.-Z., Numerical simulation of azimuth electromagnetic wave tool response based on selfadaptive FEM

Li, H., Jiang, L.-X., Jiang, Y.-R., Zhu, J.-M. \& Chen, Z.-C., Sterilization by negative and positive DC plasma with a micro discharge gap at atmospheric pressure

Li, H., Li, X. \& Wang, J., The spin Hall effect of light in moving medium

Li, H., Liu, H., Li, Y. \& Liu, Q., One-step hydrothermal fabrication and optical properties of $\mathrm{ZnO}$ nanoplate

Li, H., Wu, Y., Zeng, X., Wang, X. \& Zhao, D., Property evaluations of hydrocarbon fuels under supercritical conditions based on cubic equation of state

Li, H., Zhu, X.-F. \& Cui, C.M., Through casing weak electromagnetic wave signal detection and application

Li, H., see Chu

Li, H., see Zhang

Li, H.-N., see Feng

Li, J., see Cong

Li, J., see Dong

Li, J., see Liang

Li, J., see Liu

Li, J., see Wang

Li, J., see Zhu

Li, J.-C., Dong, Z.-W., Yang, G.-H. \& Long, C., The roles of the trading time risks on stock investment return and risks in stock price crashes

Li, J.-N., Hu, S.-L., Dong, H.-Y., Xu, X.-Y., Wang, J.F., Li, A., Wang, Q.-G. \& Li, Y.-L., The tuning effect of the electric field on the physical properties of some typical wurtzite semiconductors
B31 (2017) 1740029

B31 (2017) 1750296

B31 (2017) 1650427

B31 (2017) 1750205

B31 (2017) 1750164

B31 (2017) 1740049

B31 (2017) 1740085

B31 (2017) 1730004

B31 (2017) 1750137

B31 (2017) 1750115

B31 (2017) 1750281

B31 (2017) 1750241

B31 (2017) 1750339

B31 (2017) 1750044

B31 (2017) 1750215

B31 (2017) 1750077

B31 (2017) 1750310
Li, J.-Y., see Feng

Li, L., see Zhao

Li, L., see Zhao

Li, L.-N., Ma, C.-M., Chang, M. \& Zhang, R.-C., A quick method based on SIMPLISMA-KPLS for simultaneously selecting outlier samples and informative samples for model standardization in near infrared spectroscopy

Li, L.H., see Bi

Li, M., Xiao, X., Yang, Q. \& Yu, S., 40 Gbaud binary phase shift keying signal modulation using a substrate removed silicon modulator

Li, M., see Cai

Li, M., see Xu

Li, N., Liu, Y., Li, S., Wang, X., Qin, Y., Sun, M. \& Zhao, X., High-precision, pressure-driven pump for sub-picoliter scale quantitative injection

Li, P., see Chen

Li, P., see Dong

Li, P., see Guo

Li, Q., see Jiang

Li, Q., see Liu

Li, Q., see Yan

Li, Q.F., see Yang

Li, R., see Wang

Li, R., see Zhao

Li, S. \& Cao, D., Variable speed limit strategies' analysis with cell transmission model on freeway

Li, S., Shi, J.-D., Sun, W.-Y., Wang, D. \& Ye, L., Coherence of two-level atoms within cavity QED

Li, S., see Guo

Li, S., see Liu

Li, S., see Li

Li, S., see Sun

Li, T., Zhang, J., Ma, Y., Yu, Y. \& Zhao, Y., Effect of Ge atoms on crystal structure and optoelectronic properties of hydrogenated Si-Ge films

Li, W., see Huang
B31 (2017) 1750137

B31 (2017) 1750008

B31 (2017) 1750084

B31 (2017) 1750327

B31 (2017) 1740006

B31 (2017) 1740009

B31 (2017) 1750347

B31 (2017) 1750338

B31 (2017) 1750148

B31 (2017) 1650426

B31 (2017) 1750061

B31 (2017) 1750221

B31 (2017) 1740004

B31 (2017) 1740011

B31 (2017) 1750089

B31 (2017) 1750323

B31 (2017) 1750181

B31 (2017) 1750312

B31 (2017) 1750219

B31 (2017) 1750330

B31 (2017) 1750245

B31 (2017) 1750266

B31 (2017) 1750148

B31 (2017) 1750295

B31 (2017) 1740010

B31 (2017) 1750275 
Author Index

Li, W.-K., see Wang

Li, X., An, C., Wang, Z., Xu, C., Shi, G., Wang, J., Hou, Z. \& Xi, L., Improvement of microwave magnetic properties by inserting nonmagnetic layer

Li, X., Yin, L., Chen, W., Gao, Z. \& Liu, X., A high-resolution tunneling magneto-resistance sensor interface circuit

Li, X., see Gao

Li, X., see Kou

Li, X., see Li

Li, X., see Li

Li, X., see Song

Li, X., see Su

Li, X., see Wang

Li, X., see Zhang

Li, X.-N., see Xu

Li, X.-N., see Xu

Li, Y., Chen, W., Peeta, S., He, X., Zheng, T. \& Feng, H., An extended microscopic traffic flow model based on the spring-mass system theory

Li, Y., Sun, L., Xing, J., Ma, S., Zheng, Q. \& Liu, Y., The pressure dependence of physical properties of $\left(\mathrm{W}_{2 / 3} \mathrm{Ti}_{1 / 3}\right)_{3} \mathrm{AlC}_{2}$ and its counterpart $\mathrm{W}_{3} \mathrm{AlC}_{2}$ by first-principles calculations

Li, Y., Yang, C., Wu, L. \& Zhang, R., Electrical and optical properties of $\mathrm{Si}$ doped $\mathrm{Ga}_{2} \mathrm{O}_{3}$

Li, Y., Zhang, C.-J., Wang, T.-B., Liu, J.-T., Yu, T.B., Liao, Q.-H. \& Liu, N.H., Modulation of electromagnetic local density of states by coupling of surface phonon-polariton

Li, Y., see Chen

Li, Y., see Deng

Li, Y., see Feng

Li, Y., see Feng

Li, Y., see Liu

Li, Y., see Li

Li, Y., see Luo

Li, Y., see Zhang
B31 (2017) 1750244

B31 (2017) 1750004

B31 (2017) 1750030

B31 (2017) 1750093

B31 (2017) 1750253

B31 (2017) 1740092

B31 (2017) 1650427

B31 (2017) 1750280

B31 (2017) 1750229

B31 (2017) 1650430

B31 (2017) 1750304

B31 (2017) 1750042

B31 (2017) 1750055

B31 (2017) 1750090

B31 (2017) 1750326

B31 (2017) 1750172

B31 (2017) 1750050

B31 (2017) 1750271

B31 (2017) 1750079

B31 (2017) 1750282

B31 (2017) 1750029

B31 (2017) 1750339

B31 (2017) 1750205

B31 (2017) 1750341

B31 (2017) 1750195
Li, Y., see Zhang

Li, Y., Heat pipe cooling system of high-power threelevel explosion-proof inverter based on the loss calculation and finite element analysis

Li, Y.-L., see Li

Li, Y.-X., see Xu

Li, Y.-X., see Xu

Li, Z., see Bian

Li, Z., see Bing

Li, Z.-L., see Fang

Li, Z.-Z., Wei, Y., Zhou, H.B. \& Lu, G.-H., Effects of boron on the mechanical properties of the TiAL-Ti 3 Al alloy: A firstprinciples investigation

Li, Z.N., see Song

Liang, B., see Zhang

Liang, D., Ding, Y., Wang, N., Cai, X., Li, J., Han, L., Wang, S., Han, Y., Jia, G. \& Wang, L., Solid-state reaction synthesis for mixedphase $\mathrm{Eu}^{3+}$-doped bismuth molybdate and its luminescence properties

Liang, D., Shen, W., Zhang, C., Lu, P. \& Wang, S., Structural, electronic, vibrational and optical properties of $\mathrm{Bi}_{n}$ clusters

Liang, D., see Chen

Liang, J., see Cai

Liang, J., see Wang

Liang, J.-Q., see Zhang

Liang, R., Xi, J. \& Bao, Y., Multichannel loudness compensation method based on segmented sound pressure level for digital hearing aids

Liang, R., see $\mathrm{Xi}$

Liang, Y., see Zhao

Liang, Y., see Zhu

Liang, Z. \& Wu, X., Data hiding in halftone images with authentication ability using three-level noise-balanced error diffusion

Liang, Z., see Shen

Liao, Q.-H., see Li

Liao, Y., see Liu
B31 (2017) 1750304

B31 (2017) 1740005

B31 (2017) 1750310

B31 (2017) 1750042

B31 (2017) 1750055

B31 (2017) 1750173

B31 (2017) 1750207

B31 (2017) 1750194

B31 (2017) 1750002

B31 (2017) 1750124

B31 (2017) 1750051

B31 (2017) 1750241

B31 (2017) 1750260

B31 (2017) 1750271

B31 (2017) 1750347

B31 (2017) 1750181

B31 (2017) 1750032

B31 (2017) 1740059

B31 (2017) 1740094

B31 (2017) 1750123

B31 (2017) 1740027

B31 (2017) 1740056

B31 (2017) 1740032

B31 (2017) 1750050

B31 (2017) 1750339 
Liao, Y.-L., Zhao, Y., Zhang, X., Zhang, W. \& Chen, Z., An ultra-narrowband TEpolarization absorber with a dielectric grating and metal substrate

Liao, Y.-L., Zhao, Y., Zhang, X., Zhang, W. \& Wang, Z., Spatially and spectrally resolved ultra-narrowband TE-polarization absorber based on the guide-mode resonance

Lin, J., see Zhou

Lin, M., see Cai

Lin, R., see Chen

Lin, S., see Cai

Lin, S., see Wang

Lin, S.-X., Zhao, X.-F. \& Liu, H.-Z., Vision-based fast location of multi-bar code in any direction

Lin, Y.-N., see Yan

Lin, Y.H., Tong, C.C., Pan, Y., Liu, W.Y. \& Singh, A., Elastic properties and electronic structure of $\mathrm{Mo}_{2} \mathrm{FeB}_{2}$ alloyed with $\mathrm{Cr}$, Ni and $\mathrm{Mn}$ by firstprinciples calculations

Lin, Z.J., see Duan

Ling, W.D., Wei, P., Duan, J.Z. \& Duan, W.S., Firstprinciples study of newly synthesized nanolaminate $\mathrm{Mo}_{2} \mathrm{Ga}_{2} \mathrm{C}$

Liu, B.T., see Song

Liu, C., Zhang, H., Su, H., Li, J., Liao, Y., Jia, L. \& Li, Y., Low-temperature sintered $\mathrm{Li}_{2}\left(\mathrm{Mn}_{x} \mathrm{Ti}_{1-x}\right) \mathrm{O}_{3}$ microwave dielectric ceramics with adjustable $\tau_{f}$

Liu, C., see Cai

Liu, C., see Dai

Liu, D., Wu, L., Liu, Q., Yang, S., Zhou, R., Xie, S., Chen, J., Zhou, J. \& Chen, Z., Optical bistability effect in SPP-based metallic grating containing Kerr nonlinear medium

Liu, D., see Yang

Liu, D.-Y., Tian, B. \& Xie, X.-Y., Bound-state solu- tions, Lax pair and conservation laws for the coupled higher-order nonlinear Schrödinger equations in the birefringent or two-mode fiber

Liu, D.-Y., see Zhao

Liu, G., see Hou

Liu, G., see Yang

Liu, H., see Feng

Liu, H., see Li

Liu, H., see Wang

B31 (2017) 1750223

B31 (2017) 1750225

B31 (2017) 1750347

B31 (2017) 1740016

B31 (2017) 1750102

B31 (2017) 1750291

B31 (2017) 1740047

B31 (2017) 1750098

B31 (2017) 1750138 B31 (2017) 1750144

B31 (2017) 1750248

B31 (2017) 1750124

B31 (2017) 1750339

B31 (2017) 1750347

B31 (2017) 1750232

B31 (2017) 1750146 B31 (2017) 1750321
Liu, H., see Zhang

Liu, H., see Zhang

Liu, H.-T., Wen, Z.-Y., Xu, Y., Shang, Z.-G., Peng, J.-L. \& Tian, P., An integrated microfluidic analysis microsystems with bacterial capture enrichment and in-situ impedance detection

Liu, H.-Y., see Xie

Liu, H.-Z., see Lin

Liu, H.T., see Bi

Liu, J., see Cai

Liu, J., see Cai

Liu, J., see Liu

Liu, J., see Qiu

Liu, J., see Yan

Liu, K., Liu, Y., Liu, Y.-R., En, Y.-F. \& Li, B., Comparative study of mobility extraction methods in $p$ type polycrystalline silicon thin film transistors

Liu, K., see Wen

Liu, L., Dong, L., Li, Q. \& $\mathrm{Xu}, \mathrm{X}$. , Aluminum electrolytic capacitors for tablet PC

Liu, L., see Sun

Liu, L., see Xiao

Liu, L., see Yin

Liu, L., see Yuan

Liu, M., Wang, L., Sun, Q., Wang, W., Wang, G., Li, S., Zhang, L., Zhang, W., Hu, X., Gong, Y. \& Zhao, W., Frequencydegenerate parametric generation through IFWM effect in nanowaveguides
Liu, J.-T., see Li
B31 (2017) 1750067
B31 (2017) 1750013
B31 (2017) 1750188
B31 (2017) 1750321
B31 (2017) 1750029
B31 (2017) 1750205
B31 (2017) 1650430
B31 (2017) 1750317
B31 (2017) 1750222

B31 (2017) 1750233

B31 (2017) 1750239

B31 (2017) 1740047

B31 (2017) 1740006

B31 (2017) 1750347

B31 (2017) 1750347

B31 (2017) 1750332

B31 (2017) 1750192

B31 (2017) 1740034

B31 (2017) 1750050

B31 (2017) 1740007 B31 (2017) 1750056

B31 (2017) 1740011

B31 (2017) 1750065

B31 (2017) 1750130

B31 (2017) 1750132

B31 (2017) 1750100

B31 (2017) 1750266 
Liu, M., Yang, X., Zhao, B., Hou, J. \& Shum, P., Square array photonic crystal fiber-based surface plasmon resonance refractive index sensor

Liu, M., Yu, L. \& Han, X. Electrical characteristics of semiconductor bridge initiator-automobile airbag igniters

Liu, M.-Y., see Chen

Liu, N., Wang, W., Guo, L., Peng, T. \& Chen, X., Superconductivity in nitrogen-doped 3C-SiC from first-principles calculations

Liu, N.-H., see Li

Liu, Q., see Liu

Liu, Q., see Li

Liu, R.-S., see Shi

Liu, S., see He

Liu, S., see Tian

Liu, S., see Tian

Liu, T., Ji, L., He, G., Sun, X., Wang, F. \& Zhang, D., Ultrathin Au film on polymer surface for surface plasmon polariton waveguide application

Liu, T., see Chen

Liu, W., Geng, X. \& Xue, B., Some generalized coupled nonlinear Schrödinger equations and conservation laws

Liu, W., see Chen

Liu, W., see Wang

Liu, W., see Wen

Liu, W., High-order rogue waves of the BenjaminOno equation and the nonlocal nonlinear Schrödinger equation

Liu, W.Y., see Lin

Liu, X., Chen, Z., Zhao, Y., Shen, Y., Guo, Y., Huang, J., Min, J. \& Wang, L., The study of metal enhanced fluorescence property of $\mathrm{Ag} / \mathrm{ZnO}$ composite structure

Liu, X., Shen, L. \& Fan, H., Face recognition al- gorithm based on Gabor wavelet and locality preserving projections

Liu, X., see Gao

Liu, X., see Li

B31 (2017) 1750352

Liu, X.-W., see Di

Liu, X.-W., see Fu

Liu, X.-W., see Yin

Liu, Y., En, Y.-F. \& Fang, W.-X., Analysis of low frequency noise characteristics in $p$-type polycrystalline silicon thin film transistors

Liu, Y., see Jia

Liu, Y., see Liu

Liu, Y., see Li

B31 (2017) 1750116

B31 (2017) 1750050

B31 (2017) 1750146

B31 (2017) 1750205

B31 (2017) 1750247

B31 (2017) 1750026

B31 (2017) 1750110

B31 (2017) 1750259

B31 (2017) 1750300

B31 (2017) 1750034

Liu, Y., see Li

Liu, Y., see Wang

Liu, Y., see Xie

Liu, Y., see Zhang

Liu, Y.-R., Liu, J., Song, J.Q., Lai, P.-T. \& Yao, R.H., Indium-gallium-zincoxide thin-film transistor with a planar split dualgate structure

Liu, Y.-R., see Liu

Liu, Y.-W., see Yan

Liu, Z., see Xie

Liu, Z.H., see Yang

Lo, A., A short proof of the McCoy conjecture in higher-dimensional classical continuous models of Kac types

B31 (2017) 1750299

B31 (2017) 1750086

B31 (2017) 1750181

B31 (2017) 1750056

B31 (2017) 1750269

B31 (2017) 1750138

B31 (2017) 1750073
Lohrasby, F., see Kordani

Long, C., see Li

Long, J., see Tian

Long, Y., Zhang, X.-J. \& Wang, K., Theoretical solutions for degree distribution of decreasing random birth-and-death networks

Lou, J.Z., see Song

Lu, D., see Xie

$\mathrm{Lu}, \mathrm{F}$., $\mathrm{MoS}_{2}$-wrapped microfiber-based multi-wavelength soliton fiber laser

Lu, F., Passively harmonic mode-locked fiber laser based on $\mathrm{ReS}_{2}$ saturable absorber

Lu, G.-H., see Li

Lu, P., see Liang
B31 (2017) 1740041

B31 (2017) 1750070

B31 (2017) 1750030

B31 (2017) 1750097

B31 (2017) 1750064

B31 (2017) 1750302

B31 (2017) 1740020

B31 (2017) 1750277

B31 (2017) 1740007

B31 (2017) 1750148

B31 (2017) 1750326

B31 (2017) 1750181

B31 (2017) 1740055

B31 (2017) 1750147

B31 (2017) 1750332

B31 (2017) 1740007

B31 (2017) 1750191

B31 (2017) 1740086

B31 (2017) 1750323

B31 (2017) 1750111

B31 (2017) 1750235

B31 (2017) 1750077

B31 (2017) 1750267

B31 (2017) 1750161

B31 (2017) 1750124

B31 (2017) 1740086

B31 (2017) 1750303

B31 (2017) 1750206

B31 (2017) 1750002

B31 (2017) 1750260 
Lu, W., see Peng

Lu, X., see Wei

Lu, Y., see Bing

Luan, B., see Gao

Luo, B., see $\mathrm{Mu}$

Luo, H. \& Cheng, Y.Z., Design of an ultrabroadband visible metamaterial absorber based on three-dimensional metallic nanostructures

Luo, L., see $\mathrm{Hu}$

Luo, S., see Chen

Luo, X., see Zhao

Luo, Y., Li, Y., Guo, P., Chen, W., Tang, Y. \& Jia, Y., Band structures of onedimensional buckled Arsenene nanoribbons: Strain and quantum size modulations

Luo, Y., see Chen

Luo, Y., see Hamidnia

Lv, J. \& Xu, L., Method to acquire regions of fruit, branch and leaf from image of red apple in orchard

Lv, J., see Zhang

Lv, P., see Qiu

Lv, T. \& Feng, M., A smooth local path planning algorithm based on modified visibility graph

Ma, B., Huang, Z.X., Zu, X.D., Xiao, Q.Q. \& Jia, $\mathrm{X}$., Theoretical and experimental study on the effect of the external strong magnetic field on the shaped charge jet

Ma, C., Qi, R., Yuan, F., Chen, S. \& Zhao, H., Doping and energy dependences of thermal conductivity in cuprate superconductors

$\mathrm{Ma}, \mathrm{C}$, see $\mathrm{He}$

$\mathrm{Ma}, \mathrm{C}$. , see Teng

Ma, C.-M., see Li

Ma, H., see Jiang

Ma, J., see Jie

Ma, L., see $\mathrm{Hu}$

Ma, L., see Lang

Ma, L., see Zhao

Ma, L.X., see Song

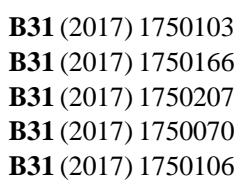

B31 (2017) 1750231

B31 (2017) 1750182

B31 (2017) 1750242

B31 (2017) 1740067

B31 (2017) 1750341

B31 (2017) 1750242

B31 (2017) 1750279

B31 (2017) 1740039

B31 (2017) 1750171

B31 (2017) 1750192

B31 (2017) 1740091

B31 (2017) 1750018

B31 (2017) 1750344

B31 (2017) 1750095

B31 (2017) 1750204

B31 (2017) 1750327

B31 (2017) 1750261

B31 (2017) 1740023

B31 (2017) 1750182

B31 (2017) 1740093

B31 (2017) 1750177

B31 (2017) 1750124
Ma, M., see Deng

Ma, M., see Wang

Ma, S., see $\mathrm{Li}$

Ma, S.-Y., see Wang

Ma, T. \& Xia, Z., An improved label propagation algorithm based on node importance and random walk for community detection

Ma, T., see Wang

Ma, W., see $\mathrm{He}$

Ma, W.-X., see Yong

Ma, Y., see Li

Mahieddine, A., see Mazouz

Mahyuddin, A., Azrina, A., Mohd Yusoff, M.Z. \& Hassan, Z., Fabrication and characterization of AlN metal-insulatorsemiconductor grown $\mathrm{Si}$ substrate

Maisnam, M., see Touthang

Maisnam, M., see Victory

Mansour, M., see Achkir

Mao, G.-Y., see Di

Mao, G.-Y., see Yu

Mao, H., see Yang

Mao, Z.Q., Zhan, X.Z. \& Chen, X., Relative importance of surface anisotropy and defects on loop shifts in antiferromagnetic nanoparticles

Mardani, R., Kazerani, M.R. \& Shahmirzaee, H., Investigating magnetic properties of $\mathrm{Co}_{68} \mathrm{Fe}_{4} \mathrm{~B}_{15} \mathrm{Si}_{13}$ amorphous alloy by molecular dynamics and DFT calculations

Mardani, R., The synthesis of $\mathrm{Ba}^{2+}$-doped multiferroic $\mathrm{BiFeO}_{3}$ nanoparticles using co-precipitation method in the presence of various surfactants and the investigation of structural and magnetic features

Mazouz, A., Bentaiba, M. \& Mahieddine, A., Coherent and squeezed states for the 3D harmonic oscillator

Mazroui, M., see Benlattar
B31 (2017) 1740088
B31 (2017) 1750181
B31 (2017) 1750326
B31 (2017) 1750198

B31 (2017) 1750162

B31 (2017) 1750329

B31 (2017) 1750015

B31 (2017) 1750276

B31 (2017) 1740010

B31 (2017) 1750019

B31 (2017) 1750313

B31 (2017) 1750066

B31 (2017) 1750314

B31 (2017) 1750183

B31 (2017) 1740035

B31 (2017) 1740074

B31 (2017) 1740024

B31 (2017) 1750197

B31 (2017) 1750094

B31 (2017) 1750169

B31 (2017) 1750019

B31 (2017) 1750037 
Md. Shareefuddin, see Sekhar

Mehdiyev, N., see Samadov

Mei, Z., see Xie

Melkikh, A.V. \& Melkikh, E.A., Can we use thermodynamics in the systems with gravity?

Melkikh, A.V., see Melkikh

Melkikh, A.V., Quantum paradoxes, entanglement and their explanation on the basis of quantization of fields

Melkikh, E.A., Melkikh, A.V. \& Tokmantsev, V.I., Nonequilibrium fluctuations in the ring of material points with gravity

Melkikh, E.A., see Melkikh

Men, S., see Yan

Meng, F., see Feng

Mi, S.-C., see Geng

Miao, M., see Bian

Miao, X., see Xu

Min, J., see Liu

Min, Y., see Zhang

Mo, H., see Fei

Mo, M., see Fang

Mo, S., see Qin

Mo, S., see Zou

Modanese, G., Generalized Maxwell equations and charge conservation censorship

Mohd Yusoff, M.Z., see Mahyuddin

Molaei, M., see Karimipour

Morsi, R.M.M., see Ahmed

Mousavi, M., see Gh. Khorrami

$\mathrm{Mu}$, D., see Fang

$\mathrm{Mu}, \mathrm{K}$., see Wang

Mu, P., Pan, W., Yan, L., Luo, B. \& Zou, X., Optimizing chaos time-delay signature in two mutuallycoupled semiconductor lasers through controlling internal parameters

Mubarak, A.A., The mechanical, optical and thermoelectric properties of $\mathrm{MCoF}_{3}(\mathrm{M}=\mathrm{K}$ and $\mathrm{Rb})$ compounds
B31 (2017) 1750180
B31 (2017) 1750134
B31 (2017) 1740055

B31 (2017) 1750272

B31 (2017) 1650425

B31 (2017) 1750007

B31 (2017) 1650425

B31 (2017) 1750272

B31 (2017) 1740034

B31 (2017) 1750029

B31 (2017) 1750001

B31 (2017) 1750173

B31 (2017) 1750075

B31 (2017) 1750073

B31 (2017) 1750304

B31 (2017) 1750243

B31 (2017) 1750194

B31 (2017) 1750337

B31 (2017) 1750250

B31 (2017) 1750052

B31 (2017) 1750313

B31 (2017) 1750297

B31 (2017) 1750278

B31 (2017) 1750175

B31 (2017) 1750249

B31 (2017) 1750202

B31 (2017) 1750106

B31 (2017) 1750033
Muhsen, M.M., see AlJawad

Najafov, A., see Samadov

Nebioğlu, M.A., Takan, T., Altan, H., Demirhan, Y., Alaboz, H., Özyüzer, L. \& Sabah, C., An indium tin oxide metasurface filter for terahertz applications: Design, fabrication, and characterization

Nian, F. \& Yao, S., Epidemic spreading on networks based on stress response

Nikkar, A., see Rouhi

Niu, J., see Wang

Nykyruy, L., see Saliy

Orudzhev, G.S., see Ismayilova

Ovchinnikov, I.V. \& Wang, K.L., Stochastic dynamics and combinatorial optimization

Ozturk, E., see Kocyigit

Özyüzer, L., see Nebioğlu

Pan, C.L., see Gong

Pan, G., see Chao

Pan, H.Y. \& Zhao, F.Q., Free polaron energy levels in $\mathrm{Al}_{y} \mathrm{Ga}_{1-y} \mathrm{~N} / \mathrm{Al}_{x} \mathrm{Ga}_{1-x} \mathrm{~N}$ triangle quantum well structures

Pan, Q.S., see Gong

Pan, R.-J., see Fang

Pan, W., see Mu

Pan, Y., see Lin

Pandey, P.C., see Dixit

Pang, S., Huo, Y., Hao, L., Sun, K., Wang, Y.J. \& Xie, Y., The sensing characteristics based on electromagneticallyinduced transparency-like response in double-sided stub and a nanodisk waveguide system

Parlak, M., see Güllü

Peeta, S., see Li

Peng, B., see Zhou

Peng, G., Lu, W., He, H. \& $\mathrm{Gu}, \mathrm{Z}$., Prevision of vehicle headway effect on urban traffic with a new carfollowing model
B31 (2017) 1750234

B31 (2017) 1750134

B31 (2017) 1750074

B31 (2017) 1750131

B31 (2017) 1750208

B31 (2017) 1750082

B31 (2017) 1750023

B31 (2017) 1750155

B31 (2017) 1750285

B31 (2017) 1750288

B31 (2017) 1750074

B31 (2017) 1750024

B31 (2017) 1750311

B31 (2017) 1750187

B31 (2017) 1750024

B31 (2017) 1750194

B31 (2017) 1750106

B31 (2017) 1750138

B31 (2017) 1750156

B31 (2017) 1750101

B31 (2017) 1750043

B31 (2017) 1750090

B31 (2017) 1750046

B31 (2017) 1750103 
Peng, H., see Zhao

Peng, H., see Zhao

Peng, J., see Dai

Peng, J.-L., see Liu

Peng, J.-Y., see Fang

Peng, P., see $\mathrm{Si}$

Peng, T., see Liu

Peng, Y., see Wang

Phanjoubam, S., see Victory

Pouresmaeeli, F., see Bordbar

Qaisar, S., see ur Rehman

Qi, L., see Zhao

Qi, R., see Ma

Qi, X.-Q., Dai, C.-J. \& Zhong, Z.-X., Optimal selection of photo-ionization and electric field ionization

Qian, Y.-S., see Zeng

Qian, Y.-S., see Zeng

Qiao, D., see Zhi

Qin, A., see Wang

Qin, G., Wang, Y., Mo, S., Fu, X., Wang, H., Xue, T., Chen, X. \& Zou, Q., Impact of various reactant concentrations on the morphology and photoluminescence property of synthetic $\mathrm{ZnO}$ nanobelts

Qin, G., see Zou

Qin, T., see Zhan

Qin, Y., see Li

Qin, Y., see Yang

Qin, Y., see Yin

Qiu, C., Cheng, H. \& Chen, S., Research of bubble flow characteristics in microfluidic chip

Qiu, C., see Cheng

Qiu, H., see Wang

Qiu, W., Liu, J., Wang, Y., Yang, Y., Lv, P. \& Jiang, Q., Saturation phenomenon research of fast light in a $\mathrm{Tm}^{3+}$-doped optical fiber at room temperature

Quan, L., see Zou

Quan, L., see Zou

Rafic, S.N., see Al-Jawad

Ramadevudu, G., see Sekhar

Ramesh, K.V., see Venkatesh

Ran, F., see Zhai

Rao, F., Chan, A.H.S. \&

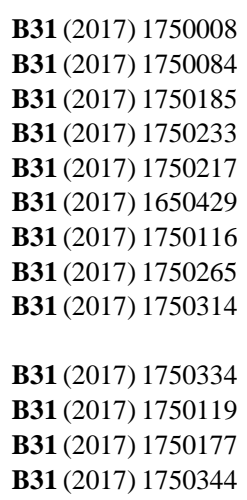

B31 (2017) 1750319

B31 (2017) 1750238

B31 (2017) 1750328

B31 (2017) 1750040

B31 (2017) 1750021

B31 (2017) 1750337
B31 (2017) 1750250
B31 (2017) 1750154
B31 (2017) 1750148
B31 (2017) 1750230
B31 (2017) 1750302

B31 (2017) 1750109

B31 (2017) 1750325

B31 (2017) 1650430

B31 (2017) 1750192

B31 (2017) 1740064

B31 (2017) 1740066

B31 (2017) 1750234

B31 (2017) 1750180

B31 (2017) 1750318

B31 (2017) 1740050
Zhu, X.-F., The influence of LED lighting on task accuracy: time of day, gender and myopia effects

Rao, F., see Chu

Rastkhadiv, M.A., see Bordbar

Rehman, Z., see Hayat

Ren, B.-Q., see Yan

Ren, J.F., see Tian

Ren, T.-L., see Wang

Ren, T.-L., see Xu

Ren, T.-L., see Xu

Ren, Z., see Chen

Rezanejad, M., see Kordani

Rong, L.-L., see Yan

Rouhi, S., Ansari, R. \& Ahmadi, M., Finite element investigation into the thermal conductivity of carbon nanotube/aluminum nanocomposites

Rouhi, S., Ansari, R. \& Nikkar, A., On the vibrational characteristics of single-walled boron nitride nanotubes/polymer nanocomposites: A finite element simulation

Ruan, X., see Zhu

Ruan, X.-X., see Fang

Ruvinskiy, M., see Saliy

Sabah, C., see Akgol

Sabah, C., see Altintas

Sabah, C., see Nebioğlu

Sabah, C., see Shawky

Sakiroglu, S., see Vatansever

Salah, M., see AbdelRahman

Saletsky, A.M., see Sidorenkov

Saletsky, A.M., see Tsysar

Saliy, Y., Ruvinskiy, M. \& Nykyruy, L., Statistics of nano-objects characteristics on the surface of PbTe: Bi condensate deposited on ceramic

Samadov, O., Alakbarov, O., Najafov, A., Samadov, S., Mehdiyev, N. \& Huseynov, E., Investigating the dielectric properties and lowfrequency relaxation process of $\mathrm{TlGaSe}_{2}$ crystals Samadov, S., see Samadov
B31 (2017) 1740019

B31 (2017) 1740021

B31 (2017) 1750228

B31 (2017) 1750316

B31 (2017) 1750191

B31 (2017) 1750335

B31 (2017) 1650430

B31 (2017) 1750042

B31 (2017) 1750055

B31 (2017) 1650426

B31 (2017) 1750235

B31 (2017) 1750089

B31 (2017) 1750053

B31 (2017) 1750208

B31 (2017) 1740054

B31 (2017) 1750217

B31 (2017) 1750023

B31 (2017) 1750176

B31 (2017) 1750274

B31 (2017) 1750074

B31 (2017) 1750072

B31 (2017) 1750071

B31 (2017) 1750255

B31 (2017) 1750289

B31 (2017) 1750142

B31 (2017) 1750023

B31 (2017) 1750134 B31 (2017) 1750134 
Sandeep Dahiya, S. \& Singh, N., Parametric excitation of optical phonons in weakly polar narrow band gap magnetized semiconductor plasmas

Sang, A., see Kou

Sangachin, E.A., see Gh. Solookinejad

Saw, S.H., see Akel

Sbiaai, K., see Benlattar

Sekhar, K.C., Hameed, A. Ramadevudu, G., Chary, M.N. \& Md. Shareefuddin Physical and spectroscopic studies on manganese ions in lead halo borate glasses

Settaouti, A., see Drize

Sh. Ismael, see Akel

Shah, Z.A., see Hayat

Shahmirzaee, H., see Mardani

Shang, J., see Zhang

Shang, Z., Li, D., Yin, S. \& Wang, S., Preparation of high-oriented molybdenum thin films using DC reactive magnetronsputtering

Shang, Z.-G., see Liu

Shao, J., see Wang

Shao, S., see Sun

Shao, Z., see Wang

Shawky, N., Taha, S.A.A. Altan, H. \& Sabah, C., Single- and double-sided sensor applications of metamaterials based on square-ring and diamond resonators for terahertz region

Shen, H. \& Tan, Y.-S., Vehicle handling and stability control by the cooperative control of 4WS and DYC

Shen, H., Liang, Z. \& Cheng, B., Improvement of heterogeneous deformation measurement in digital image correlation

Shen, H., see Chen

Shen, J., see $\mathrm{He}$

Shen, K., see Yang

Shen, L., see Liu

Shen, W., see Liang
B31 (2017) 1750294

B31 (2017) 1750253

B31 (2017) 1750322

B31 (2017) 1750167

B31 (2017) 1750037

B31 (2017) 1750180

B31 (2017) 1750165

B31 (2017) 1750167

B31 (2017) 1750316

B31 (2017) 1750094

B31 (2017) 1750203

B31 (2017) 1750059

B31 (2017) 1750233

B31 (2017) 1750136

B31 (2017) 1750049

B31 (2017) 1750181

B31 (2017) 1750072

B31 (2017) 1740090

B31 (2017) 1740032

B31 (2017) 1750242

B31 (2017) 1750240

B31 (2017) 1740024

B31 (2017) 1740041

B31 (2017) 1750260
Shen, Y., see Deng

Shen, Y., see Liu

Shen, Y.-J., see Guo

Shen, Y.-Z., see Li

Sheng, F., see Yao

Sheng, X., see $\mathrm{Hu}$

Shi, G., see Li

Shi, G.-J., Wang, J.-G., Hou, Z.-Y., Wang, Z. \& Liu, R.S., Simulation study of the effect of strain rate on the mechanical properties and tensile deformation of gold nanowire

Shi, H., see Zheng

Shi, J., see Song

Shi, J.-D., see Li

Shi, J.-P., see Di

Shi, J.P., Prediction study on the degeneration of lithium-ion battery based on fuzzy inference system

Shi, L., see Cai

Shi, W., Zhang, X., Zou, X. \& Han, W., Deep neural network and noise classification-based speech enhancement

Shi, X., see Wang

Shi, Z., see Wang

Shin, H., see ur Rehman

Shou, G.-C., see Jin

Shum, P., see Liu

Si, F.J., Hu, W., Tang, F.L., Cheng, Y.W. \& Xue, H.T., Electronic properties and lattice configurations of $\mathrm{Au} / \mathrm{CH}_{3} \mathrm{NH}_{3} \mathrm{PbI}_{3}$ interface

Si, Y., Yang, H.-M., Wu, H.Y., Huang, W.-Q., Yang, K., Peng, P. \& Huang, G.-F., Tuning the neargap electronic structure of $\mathrm{Cu}_{2} \mathrm{O}$ by anion-cation codoping for enhanced solar energy conversion

Si, Y., see Gao

Sidorenkov, A.V., Kolesnikov, S.V. \& Saletsky, A.M., Graphene on $\mathrm{Cu}(111)$ at the nonzero temperatures: molecular dynamic simulation

Singh, A., see Lin
B31 (2017) 1750079

B31 (2017) 1750073

B31 (2017) 1750256

B31 (2017) 1740029

B31 (2017) 1740075

B31 (2017) 1750182

B31 (2017) 1750004

B31 (2017) 1750247

B31 (2017) 1740044

B31 (2017) 1750124

B31 (2017) 1750330

B31 (2017) 1740035

B31 (2017) 1740083 B31 (2017) 1750347

B31 (2017) 1740096

B31 (2017) 1750265

B31 (2017) 1750062

B31 (2017) 1750119

B31 (2017) 1740095

B31 (2017) 1750352

B31 (2017) 1750199

B31 (2017) 1650429

B31 (2017) 1740002

B31 (2017) 1750289

B31 (2017) 1750138 
Singh, N., see Sandeep

Sitnikov, I.I., see Tsysar

Smith, A.D., Ukogu, O.A., Devenica, L.M., White, E.D. \& Carter, A.R., Optical methods for measuring DNA folding

Soibam, I., see Devi

Sokmen, I., see Vatansever

Soldatov, A.V., Broadband EM radiation amplification by means of a monochromatically driven two-level system

Song, A.Y., Dai, X.H., Song, J.M., Ge, D.Y., Shi, J., Fang, X.Y., Li, Z.N., Wang, S.J., Yan, X.B., Guo, J.X., Lou, J.Z., Ma, L.X. \& Liu, B.T., Investigation of interface and oxygen vacancy of $\mathrm{Pt} /$ $\mathrm{Ba}_{0.6} \mathrm{Sr}_{0.4} \mathrm{TiO}_{3} / \mathrm{MgO}$ interdigital capacitor through sequent low and high temperature annealings

Song, C., see Ding

Song, G., see Chen

Song, H., see Cai

Song, H., see Gao

Song, J., see Ye

Song, J.-Q., see Liu

Song, J.M., see Song

Song, K., see Gong

Song, L., see Zhang

Song, S., Song, X. \& Balsera, I.T., Mixed $H_{\infty}$ and passive projective synchronization for fractional-order memristor-based neural networks with time delays via adaptive sliding mode control

Song, S., see Chen

Song, T., Li, C. \& Li, X., Gauge transformations of the multi-component BKP and CKP hierarchies

Song, X., see Song

Song, Z., see Zhang

Stamov, G. \& Stamova, I., On stable integral manifolds for impulsive Kolmogorov systems of fractional order

Stamova, I., see Stamov
B31 (2017) 1750294
B31 (2017) 1750142

B31 (2017) 1730001

B31 (2017) 1750236

B31 (2017) 1750071

B31 (2017) 1750027

B31 (2017) 1750124

B31 (2017) 1750149

B31 (2017) 1650426

B31 (2017) 1750347

B31 (2017) 1750093

B31 (2017) 1750333

B31 (2017) 1750332

B31 (2017) 1750124

B31 (2017) 1750286

B31 (2017) 1850038

B31 (2017) 1750160 B31 (2017) 1750086

B31 (2017) 1750280

B31 (2017) 1750160

B31 (2017) 1750032

B31 (2017) 1750168

B31 (2017) 1750168
Su, F., Dong, H., Jia, L. \& Sun, X., On urban road traffic state evaluation index system and method

$\mathrm{Su}, \mathrm{H}$., see Liu

$\mathrm{Su}$, J.-J., see Jia

Su, J.-J., see Wang

$\mathrm{Su}, \mathrm{W}$., see Sun

Su, X., Cui, H., Ju, W., Yong, Y. \& Li, X., Firstprinciples investigation of $\mathrm{MoS}_{2}$ monolayer adsorbed on $\mathrm{SiO}_{2}(0001)$ surface

$\mathrm{Su}$, Y., see Zhao

Sun, B., see Zhang

Sun, D., see Li

Sun, J., see Wang

Sun, K., see Pang

Sun, L., Gao, Y., Yoshida, K., Yano, T. \& Wang, W., Prediction on structural, mechanical and thermal properties of $\mathrm{Al}_{4} \mathrm{SiC}_{4}, \mathrm{Al}_{4} \mathrm{C}_{3}$ and $4 \mathrm{H}-\mathrm{SiC}$ under high pressure by first-principles calculation

Sun, L., see $\mathrm{Li}$

Sun, M., Zheng, Y., Zhang, L., Zhao, L. \& Zhang, B., Influence of heat treatment on hole transfer dynamics in core-shell quantum dot/organic hole conductor hybrid films

Sun, M., see Li

Sun, N. \& Gersappe, D., Simulation of diffuse-charge capacitance in electric double layer capacitors

Sun, Q., see Liu

Sun, W.-Y., see Li

Sun, X., see Cheng

Sun, X., see Liu

Sun, X., see Su

Sun, X.-X., Li, C., Hou, Q.Y. \& Zhang, Y., Phase transition and electronic properties of $\mathrm{SbI}_{3}$ : Firstprinciples calculations

Sun, Y., Dai, M., Shao, S. \& Su, W., The entire mean weighted firstpassage time on infinite families of weighted tree networks
B31 (2017) 1650428

B31 (2017) 1750339

B31 (2017) 1750129

B31 (2017) 1750012

B31 (2017) 1750049

B31 (2017) 1750229

B31 (2017) 1730003

B31 (2017) 1750087

B31 (2017) 1750085

B31 (2017) 1750136

B31 (2017) 1750101

B31 (2017) 1750080 B31 (2017) 1750326

B31 (2017) 1750218

B31 (2017) 1750148

B31 (2017) 1650431

B31 (2017) 1750266

B31 (2017) 1750330

B31 (2017) 1750325

B31 (2017) 1750300

B31 (2017) 1650428

B31 (2017) 1750200

B31 (2017) 1750049 
Author Index

Sun, Y., Li, S. \& Jiao, Z., Acoustic response of multi-stopband local resonance plate

Sun, Y., Tian, B., Wu, X.-Y., Liu, L. \& Yuan, Y.-Q., Dark solitons for a variable-coefficient higherorder nonlinear Schrödinger equation in the inhomogeneous optical fiber

Sun, Y., see Wu

Sun, Y., see Xiao

Sy, P.-V., see Kim

Taha, S.A.A., see Shawky

Takan, T., see Nebioğlu

Tan, C., see Dai

Tan, C., see Zhou

Tan, J.-G., see Guo

Tan, M.-S., see Ding

Tan, Y.-S., see Shen

Tang, F.L., see $\mathrm{Si}$

Tang, H.-G., see Leng

Tang, J., see Chen

Tang, L., see Zhi

Tang, T.-Q., see Xiao

Tang, T.-Q., see Xiao

Tang, X., see Zhang

Tang, Y., see Luo

Tang, Z., see Zhang

Tao, X., see Yang

Tao, X., see Zou

Tavassoly, M.K., see Faraji

Teng, Y., Gao, H., Ma, C., Yuan, F. \& Zhao, H., The non-Drude type of optical conductivity in cuprates

Thakur, A.K., see Gargama

Tian, B., see Chai

Tian, B., see Liu

Tian, B., see Sun

Tian, B., see Wu

Tian, B., see Xiao

Tian, B., see Yin

Tian, B., see Yuan

Tian, B., see Yu

Tian, B., see Zhao

Tian, J., Zhang, C., Zhang, L., Zheng, M. \& Liu, S., Corresponding statebased correlations for the temperature-dependent surface tension of saturated hydrocarbons

Tian, J., Zhang, L. \& Zheng,
M., A new correlation in predicting temperaturedependent viscosity of saturated liquids

B31 (2017) 1750295

Tian, J., Zheng, M., Yi, H., Zhang, L. \& Liu, S., Corresponding state-based correlations for the surface tension of saturated fluids

Tian, J., see Yong

B31 (2017) 1750065

B31 (2017) 1750122

B31 (2017) 1750130

B31 (2017) 1750081

B31 (2017) 1750072

B31 (2017) 1750074

B31 (2017) 1750232

B31 (2017) 1750184

B31 (2017) 1750256

B31 (2017) 1750252

B31 (2017) 1740090

B31 (2017) 1750199

B31 (2017) 1740053

B31 (2017) 1750271

B31 (2017) 1750040

B31 (2017) 1750121

B31 (2017) 1750324

B31 (2017) 1730004

B31 (2017) 1750341

B31 (2017) 1750195

B31 (2017) 1740024

B31 (2017) 1750250

B31 (2017) 1750038

B31 (2017) 1750204

B31 (2017) 1750301

B31 (2017) 1750135

B31 (2017) 1750067

B31 (2017) 1750065

B31 (2017) 1750122

B31 (2017) 1750130

B31 (2017) 1750132

B31 (2017) 1750100

B31 (2017) 1750258

B31 (2017) 1750013

B31 (2017) 1750259
Tian, L., see Zhu

Tian, M., Wang, X., Dong, Z., Zhu, G., Long, J., Dai, D. \& Zhang, Q., Cascading failures in interdependent modular networks with partial random coupling preference

Tian, M., see Dong

Tian, P., see Liu

Tian, S.-F., see Dong

Tian, S.-F., see Yan

Tian, S.-F., see Zou

Tian, W., see Chen

Tian, Y., see Hou

Tian, Y., see Wang

Tian, Y.L., Hua, H.L., Yue, W.W., Chen, M.N., Hu, G.C., Ren, J.F. \& Yuan, X.B., Adsorption properties of chloroform molecule on graphene: Experimental and firstprinciples calculations

Tie, S., see Chen

Tie, S., see Gao

Tie, S., see Zhan

Tokmantsev, V.I., see Melkikh

Tong, C.C., see Lin

Touthang, J. \& Maisnam, M., Structural and electrical studies of sol-gel synthesized nanocrystalline hexagonal yttrium iron manganite ceramics

Trimper, S., see Apostolov

Tsysar, K.M., Kolesnikov, S.V., Sitnikov, I.I. \& Saletsky, A.M., Ab initio investigation of supported $\mathrm{Au}-$ Mn nanowires

Turgut, G., see Kocyigit

Ukogu, O.A., see Smith

Unal, E., see Akgol
B31 (2017) 1750014

B31 (2017) 1750110

B31 (2017) 1750276

B31 (2017) 1740077

B31 (2017) 1750267

B31 (2017) 1750112

B31 (2017) 1750233

B31 (2017) 1750281

B31 (2017) 1750350

B31 (2017) 1750348

B31 (2017) 1750113

B31 (2017) 1750188

B31 (2017) 1750202

B31 (2017) 1750335

B31 (2017) 1740001

B31 (2017) 1740002

B31 (2017) 1740003

B31 (2017) 1650425

B31 (2017) 1750138

B31 (2017) 1750066 B31 (2017) 1750351

B31 (2017) 1750142

B31 (2017) 1750288

B31 (2017) 1730001

B31 (2017) 1750176 
Unal, E., see Altintas ur Rehman, J., Qaisar, S., Jeong, Y. \& Shin, H., Security of a control key in quantum key distribution

Varshney, D., see Yadav

Vatansever, Z.D., Sakiroglu, S. \& Sokmen, I., Effects of a scattering center on the ground-state energy of quantum-dot lithium

Venkatesh, D. \& Ramesh, K.V., Structural and electrical properties of $\mathrm{Cu}$ doped Ni-Zn nanocrystalline ferrites for MLCI applications

Victory, M., Maisnam, M. \& Phanjoubam, S., Influence of sintering technique on the characteristics of $\mathrm{Li}_{-}$ Co nanoferrites

Vinh, L.T., see Yen

Volkán-Kacsó, S., The elastic transfer model of angular rate modulation in $\mathrm{F}_{1}$-ATPase stalling and controlled rotation experiments

Vovk, R.V., see Boiko

Wan, T., see Zhu

Wang, A.M., see Xie

Wang, C., Ji, Z. \& Wang, Y., Multiobjective flexible job shop scheduling problem using variable neighborhood evolutionary algorithm

Wang, C., see Geng

Wang, C., see Wang

Wang, C.-Q., Zhou, J. \& Zhang, Z.-M., Generating squeezed states of a nanomechanical resonator via a charge qubit in a hybrid system

Wang, D., see Li

Wang, D., see Wang

Wang, D., see Zhao

Wang, D., see Zhou

Wang, F., see Liu

Wang, F., see Yan

Wang, G., Liu, H., Li, X., Qiu, H., Yang, Y. \& Ren, T.-L., Novel on-chip spiral
B31 (2017) 1750274

B31 (2017) 1750119 B31 (2017) 1750153

B31 (2017) 1750071

B31 (2017) 1750318

B31 (2017) 1750314 B31 (2017) 1750036

B31 (2017) 1730002
B31 (2017) 1750179
B31 (2017) 1740077
B31 (2017) 1750214

B31 (2017) 1740072

B31 (2017) 1750001

B31 (2017) 1750329

B31 (2017) 1750343

B31 (2017) 1750330

B31 (2017) 1750265

B31 (2017) 1730003

B31 (2017) 1750046

B31 (2017) 1750300

B31 (2017) 1850069 inductors with back hollow structure

Wang, G., Wang, D., Shi, X. \& Peng, Y., Firstprinciples study of the electronic structure and thermoelectric properties of $\mathrm{LaOBiCh}_{2}(\mathrm{Ch}=\mathrm{S}, \mathrm{Se})$

Wang, G., see Deng

Wang, G., see Huang

Wang, G., see Liu

Wang, G., see Wang

Wang, G., see Zhao

Wang, H., see Li

Wang, H., see Qin

Wang, H., see Wei

Wang, H., see Yan

Wang, H., see Yao

Wang, H., see Zeng

Wang, H., see Zou

Wang, H.-D., see Wang

Wang, J., Cheng, H., Gao, L., Wang, X., Duan, R. \& Li, J., Dielectric properties of $x \mathrm{Na}_{0.5} \mathrm{Bi}_{0.5} \mathrm{TiO}_{3}-(1-x)$

$\mathrm{Ba}_{0.66} \mathrm{Mg}_{0.04} \mathrm{Sr}_{0.3} \mathrm{TiO}_{3}$ composite ceramics

Wang, J., Cheng, J.-T., Ma, S.-Y. \& Wang, H.-D., Adsorption of self-assembled monolayer on $\mathrm{Cu}(111)$ : First-principles study

Wang, J., Yin, C., Zhu, M., Sun, J., Yi, K. \& Shao, J., Wide angle and broadband perfect absorber with compact multilayer structures

Wang, J., Zhang, J., Tian, Y., Mu, K., Fan, C., Chen, S. \& Ding, P., Fano resonances in nanorod dimers antenna

Wang, J., see Li

Wang, J., see Li

Wang, J., see Wang

Wang, J., see Xie

Wang, J., see Yuan

Wang, J., see Zhang

Wang, J., see Zhou

Wang, J.-B., see Xu

Wang, J.-B., see Xu

Wang, J.-F., see $\mathrm{Li}$

Wang, J.-G., see Shi

Wang, J.-X., see Wang

Wang, J.-X., see Wang
B31 (2017) 1650430

B31 (2017) 1750265

B31 (2017) 1750079

B31 (2017) 1750275

B31 (2017) 1750266

B31 (2017) 1750193

B31 (2017) 1750177

B31 (2017) 1750085

B31 (2017) 1750337

B31 (2017) 1750166

B31 (2017) 1750211

B31 (2017) 1750076

B31 (2017) 1750238

B31 (2017) 1750250

B31 (2017) 1750198

B31 (2017) 1750044

B31 (2017) 1750198

B31 (2017) 1750136

B31 (2017) 1750202

B31 (2017) 1650427

B31 (2017) 1750004

B31 (2017) 1750181

B31 (2017) 1740086

B31 (2017) 1750060

B31 (2017) 1750032

B31 (2017) 1750225

B31 (2017) 1750042

B31 (2017) 1750055

B31 (2017) 1750310

B31 (2017) 1750247

B31 (2017) 1750104

B31 (2017) 1750244 
Wang, K., see Long

Wang, K.L., see Ovchinnikov

Wang, L., see Dai

Wang, L., see Liang

Wang, L., see Liu

Wang, L., see Liu

Wang, L., see $\mathrm{Li}$

Wang, L., see Wang

Wang, L., see Zhao

Wang, L.-F., Du, M.-M. \& Ye, L., Protecting quantum coherence in an open system under non-inertial frames

Wang, L.-L., see Cui

Wang, L.-Q., see Yan

Wang, N., see Liang

Wang, P., Gao, Z., Cheng, D., Xu, D. \& Niu, J., Effect of Ni-P alloy coating on microstructures and properties of vacuum brazed joints of $\mathrm{SiCp} / \mathrm{Al}$ composites

Wang, P., Xiao, S.-H., Chen, L. \& Yang, G., Analytical study on the generalized Davydov model in the alpha helical proteins

Wang, P., see Chen

Wang, P., Bilinear form and soliton solutions for the fifth-order Kaup-Kupershmidt equation

Wang, Q. \& Wang, W.-G., Probing quantum critical points by Fisher information at finite temperature

Wang, Q., see Cai

Wang, Q., see Wang

Wang, Q., see Zhang

Wang, Q.-G., see Li

Wang, R., see Zhou

Wang, R.-Z., see Fang

Wang, S., see Deng

Wang, S., see Liang

Wang, S., see Liang

Wang, S., see $\mathrm{Li}$

Wang, S., see Shang

Wang, S., see Yan

Wang, S.-H., see Wang

Wang, S.J., see Song

Wang, T., see Cong

Wang, T.-B., see Li
B31 (2017) 1750161
B31 (2017) 1750285
B31 (2017) 1750232
B31 (2017) 1750241
B31 (2017) 1750266
B31 (2017) 1750073
B31 (2017) 1750085
B31 (2017) 1750062
B31 (2017) 1750177

B31 (2017) 1750336

B31 (2017) 1750108

B31 (2017) 1750191

B31 (2017) 1750241

B31 (2017) 1750082

B31 (2017) 1750178 B31 (2017) 1750086

B31 (2017) 1750057

B31 (2017) 1750107

B31 (2017) 1750347

B31 (2017) 1750354

B31 (2017) 1750222

B31 (2017) 1750310

B31 (2017) 1750225

B31 (2017) 1750217

B31 (2017) 1750079

B31 (2017) 1750241

B31 (2017) 1750260

B31 (2017) 1750270

B31 (2017) 1750059

B31 (2017) 1850069

B31 (2017) 1750329

B31 (2017) 1750124

B31 (2017) 1750115

B31 (2017) 1750050
Wang, T.-J., see Geng

Wang, T.-J., see Wang

Wang, W., Bao, X., Chen, L., Chen, T., Wang, G. \& Yuan, J., High photon detection efficiency single photon avalanche diode in $0.18 \mu \mathrm{m}$ standard CMOS process

Wang, W., Hu, X. \& Wei, X., $0^{\circ}$ to $90^{\circ}$ arbitrary controllable optical activity with an array of $L$-shaped silver holes inside a silica cube

Wang, W., see Liu

Wang, W., see Liu

Wang, W., see Sun

Wang, W.-G., see Wang

Wang, X. \& Huang, C., Noise-enhanced CVQKD with untrusted source

Wang, X., Wang, Q., Dong, G., Hao, Y., Lei, M. \& Bi, K., Multi-band terahertz metasurface absorber

Wang, X., see Hamidnia

Wang, X., see Kou

Wang, X., see Li

Wang, X., see Li

Wang, X., see Tian

Wang, X., see Wang

Wang, X., see Xie

Wang, X., see Ye

Wang, X.-Q., The influence on the average number of optical phonons in an asymmetrical Gaussian potential polar slab of semiconductors

Wang, X.H., Shi, Z. \& Wang, L., Effects of sintering atmospheres on phase transformation, oxygen vacancy and photoabsorption behaviors of highly Fe-doped titania crystals

Wang, Y. \& Ji, Z., Energy efficiency quantitative analysis method of discrete manufacturing system

Wang, Y., Huang, S. \& Ji, Z., Operation management of daily economic dispatch using novel hybrid particle swarm optimization
B31 (2017) 1750001

B31 (2017) 1750329

B31 (2017) 1750193

B31 (2017) 1750309

B31 (2017) 1750266

B31 (2017) 1750116

B31 (2017) 1750080

B31 (2017) 1750107

B31 (2017) 1750143

B31 (2017) 1750354

B31 (2017) 1750279

B31 (2017) 1750253

B31 (2017) 1750164

B31 (2017) 1750148

B31 (2017) 1750267

B31 (2017) 1750044

B31 (2017) 1750239

B31 (2017) 1750047

B31 (2017) 1750141

B31 (2017) 1750062

B31 (2017) 1740071 
and gravitational search algorithm with hybrid mutation strategy

Wang, Y., Qin, A., Chu, L., Deng, Z., Ding, X. \& Guan, L., A nucleation and growth model of silicon nanoparticles produced by pulsed laser deposition via Monte Carlo simulation

Wang, Y., see Chen

Wang, Y., see Chen

Wang, Y., see Deng

Wang, Y., see Huang

Wang, Y., see $\mathrm{Hu}$

Wang, Y., see Qin

Wang, Y., see Qiu

Wang, Y., see Wang

Wang, Y., see Wang

Wang, Y., see Xu

Wang, Y., see Xu

Wang, Y., see Zhang

Wang, Y., see Zhang

Wang, Y., see Zhang

Wang, Y., see Zhao

Wang, Y., see Zou

Wang, Y.-J., see Pang

Wang, Y.-J., see Zhu

Wang, Y.-L., Ga, Y.-T., Jia, S.-L., Deng, G.-F. \& Hu, W.-Q., Solitons for a (2+1)-dimensional variablecoefficient BogoyavlenskyKonopelchenko equation in a fluid

Wang, Y.-L., Gao, Y.-T., Jia, S.-L., Lan, Z.-Z., Deng, G.-F. \& Su, J.-J., Solitons and integrability for a $(2+1)$-dimensional generalized variable-coefficient shallow water wave equation

Wang, Y.-Q., Chu, X.-J., Zhou, C.-F., Jia, B., Lin, S., Wu, Z.-H., Zhu, H.-B. \& Gao, Z.-Y., Evolvement law of a macroscopic traffic model accounting for density-dependent relaxation time

Wang, Y.-Q., Yan, B.-W., Zhou, C.-F., Chu, X.-J., Zhang, D.-C., Li, W.-K., Wang, J.-X., Fang, C.-H.,

\section{B31 (2017) 1740099}

B31 (2017) 1750021

B31 (2017) 1750262

B31 (2017) 1740098

B31 (2017) 1740070

B31 (2017) 1740073

B31 (2017) 1750182

B31 (2017) 1750337

B31 (2017) 1750192

B31 (2017) 1740072

B31 (2017) 1740057

B31 (2017) 1740058

B31 (2017) 1740100

B31 (2017) 1750305

B31 (2017) 1750051

B31 (2017) 1740087

B31 (2017) 1750312

B31 (2017) 1750250

B31 (2017) 1750101

B31 (2017) 1740027

B31 (2017) 1750216

B31 (2017) 1750012

B31 (2017) 1750291
Jia, B. \& Gao, Z.-Y., Theoretical analysis of bifurcations in a microscopic traffic model accounting for optimal velocity

Wang, Y.-Q., Zhou, C.-F., Jia, B. \& Zhu, H.-B., Reliability analysis of degradable networks with modified BPR

Wang, Y.-Q., Zhou, C.-F., Yan, B.-W., Zhang, D.-C., Wang, J.-X., Jia, B., Gao, Z.-Y. \& Wu, Q.-S., Theoretical analysis of a hybrid traffic model accounting for safe velocity

Wang, Y.X., see Zhang

Wang, Z., Li, R., Shao, Z., Ma, M., Liang, J., Liu, W., Wang, J. \& Liu, Y., Adaptive Harris corner detection algorithm based on iterative threshold

Wang, Z., Wu, D., Wang, Y. \& Ji, Z., Stochastic gradient identification algorithm for nonlinear system modeling in wind power curtailment prediction

Wang, Z., see Deng

Wang, Z., see Liao

Wang, Z., see $\mathrm{Li}$

Wang, Z., see Shi

Wang, Z., see Yang

Wang, Z., see Yin

Wang, Z.-X., Ma, T., Wang, S.-H., Wang, T.-J. \& Wang, C., Dynamics of coherence under Markovian and nonMarkovian environments

Watanabe, T., see Zhao

Wei, H., see Wei

Wei, L.-F., see Zhang

Wei, P., see Ling

Wei, Q., see Yang

Wei, Q., see Yang

Wei, S.-C., see Zhu

Wei, W.-H., Effects of chemical composition and mean coordination number on glass transitions in $\mathrm{Ge}-\mathrm{Sb}$ Se glasses

Wei, X., see Wang

Wei, X., see Yao
B31 (2017) 1750244

B31 (2017) 1750353

B31 (2017) 1750104

B31 (2017) 1750283

B31 (2017) 1750181

B31 (2017) 1740057

B31 (2017) 1740088

B31 (2017) 1750223

B31 (2017) 1750004

B31 (2017) 1750247

B31 (2017) 1750091

B31 (2017) 1750150

B31 (2017) 1750329

B31 (2017) 1740067

B31 (2017) 1750166

B31 (2017) 1750032

B31 (2017) 1750248

B31 (2017) 1750201

B31 (2017) 1750349

B31 (2017) 1740027

B31 (2017) 1750342

B31 (2017) 1750309

B31 (2017) 1740075 
Wei, X.-T., see Zeng

Wei, X.-T., see Zeng

Wei, Y., Wang, H., Lu, X., Fan, X. \& Wei, H., Effects of element doping on electronic structures and optical properties in cubic boron nitride from firstprinciples

Wei, Y., see Li

Wei, Y., see Zhang

Wei, Y., see Zhan

Wen, J., Liu, W., Liu, K., Fu, H. \& Jia, Z., Research and analysis on the broadband dispersion tailoring of the sub-micro siliconon-insulator waveguides

Wen, L., see Yan

Wen, L., see Yao

Wen, Y.-N., Gao, P.-F., Chen, X., Xia, M.-G., Zhang, Y. \& Zhang, S.-L., Widthdependent structural stability and magnetic properties of monolayer zigzag $\mathrm{MoS}_{2}$ nanoribbons

Wen, Z., see Gu

Wen, Z.-Y., see Liu

Wesselinowa, J.M., see Apostolov

Wesselinowa, J.M., see Apostolov

White, E.D., see Smith

Wu, C., see Deng

Wu, D. \& Zhang, X., Design of LPV fault-tolerant controller for pitch system of wind turbine

Wu, D., see Wang

Wu, F., Zhu, X. \& Jiang, X., Pentagram star pattern identification algorithm applied in three-head star sensors

$\mathrm{Wu}, \mathrm{F}$, see Gao

Wu, F., see Xiang

$\mathrm{Wu}, \mathrm{F}$, see $\mathrm{Zhu}$

Wu, H. \& Yao, Y., Analytic solutions and their dynamics of atomic-molecular Bose-Einstein condensates with time- and spacemodulated nonlinearities

Wu, H.-Y., see Si
B31 (2017) 1750238
B31 (2017) 1750328

B31 (2017) 1750166

B31 (2017) 1750002

B31 (2017) 1750087

B31 (2017) 1750154

B31 (2017) 1750056

B31 (2017) 1750211

B31 (2017) 1750076

B31 (2017) 1750017

B31 (2017) 1750048

B31 (2017) 1750233

B31 (2017) 1750351

B31 (2017) 1750009

B31 (2017) 1730001

B31 (2017) 1740070

B31 (2017) 1740068

B31 (2017) 1740057

B31 (2017) 1740046

B31 (2017) 1750093

B31 (2017) 1740038

B31 (2017) 1740037

B31 (2017) 1650372 B31 (2017) 1650429
Wu, J.H., see $\mathrm{Hou}$

Wu, L., see Chen

Wu, L., see Liu

$\mathrm{Wu}, \mathrm{L}$., see $\mathrm{Li}$

Wu, Q.-S., see Wang

$\mathrm{Wu}, \mathrm{W}$., see $\mathrm{Bi}$

Wu, W.-F. \& Fan, H.-Y., Thermo vacuum state for Gaussian-enhanced chaotic light

Wu, X., see Liang

Wu, X.-Y., Tian, B., Chai, H.P. \& Sun, Y., Rogue waves and lump solutions for a $(3+1)$-dimensional generalized B-type KadomtsevPetviashvili equation in fluid mechanics

Wu, X.-Y., see Sun

Wu, X.-Y., see Xiao

Wu, X.-Y., see Zhao

Wu, Y., Xiong, X. \& Zhang, Y., Effects of convincing power and neutrality on minority opinion spreading

Wu, Y., see $\mathrm{Li}$

Wu, Z.-H., see Wang

$\mathrm{Xi}$, B., see Guo

Xi, J., Liang, R. \& Fei, X., An algorithm of improving speech emotional perception for hearing aid

$\mathrm{Xi}$, J., see Liang

$\mathrm{Xi}, \mathrm{L}$., see $\mathrm{Li}$

Xia, M.-G., see Wen

Xia, Y.-Q., see Xie

Xia, Y.-Q., see Xie

Xia, Z., see Ma

Xiang, R., Zhu, X. \& Wu, F., Single image haze removal based on two steps

Xiang, R., see Zhu

Xiao, H., Huang, H.-J. \& Tang, T.-Q., Analysis of energy consumption and emission of the heterogeneous traffic flow consisting of traditional vehicles and electric vehicles

Xiao, H., Huang, H.-J. \& Tang, T.-Q., Impacts of road conditions on the energy consumption of electric vehicular flow
B31 (2017) 1750251

B31 (2017) 1750271

B31 (2017) 1750146

B31 (2017) 1750172

B31 (2017) 1750104

B31 (2017) 1750041

B31 (2017) 1750151

B31 (2017) 1740056

B31 (2017) 1750122

B31 (2017) 1750065

B31 (2017) 1750130

B31 (2017) 1750013

B31 (2017) 1750058

B31 (2017) 1750164

B31 (2017) 1750291

B31 (2017) 1750256

B31 (2017) 1740094

B31 (2017) 1740059

B31 (2017) 1750004

B31 (2017) 1750017

B31 (2017) 1750105

B31 (2017) 1750239

B31 (2017) 1750162

B31 (2017) 1740038

B31 (2017) 1740037

B31 (2017) 1750324

B31 (2017) 1750121 
Xiao, J., see Xiong

Xiao, J., see Zhao

Xiao, J., see Zhao

Xiao, Q.Q., see Ma

Xiao, S., see Yao

Xiao, S.-H., see Wang

Xiao, X., see Li

Xiao, Z.-J., Tian, B., Wu, X.-Y., Liu, L. \& Sun, Y., Soliton interactions of a $(2+1)$-dimensional nonlinear Schrödinger equation in a nonlinear photonic quasicrystal or Kerr medium

Xie, A.-G., Xia, Y.-Q., Wang, X., Liu, H.-Y. \& Cheng, S., Formulae for maximum yield and mean escape depth of secondary electrons emitted from metals

Xie, A.-G., Zhon, K., Zhao, D.-L. \& Xia, Y.-Q., Formulae for low-energy secondary electron yield from different kinds of emitters as a function of measurable variables

Xie, D., Xu, C. \& Wang, A.M., Gravitational time dilation induced decoherence in spontaneous emission

Xie, J., Liu, Y., Zeng, X., Zhang, W. \& Mei, Z., A Bayesian network model for predicting type 2 diabetes risk based on electronic health records

Xie, J., Zeng, X., Lu, D., Liu, Z. \& Wang, J., MHC2NNZ: A novel peptide binding prediction approach for HLA DQ molecules

Xie, S., see Liu

Xie, X.-Y., see Liu

Xie, X.-Y., see Yuan

Xie, Y., see Pang

Xie, Z., see Cai

Xin, F., see Chen

Xin, H., see Gao

Xing, J., see Li

Xing, L., Xu, X., Guan, J. \& Dong, X., Trade broker-

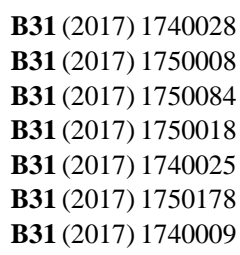

B31 (2017) 1750130

B31 (2017) 1750239

B31 (2017) 1750105

B31 (2017) 1750214

\section{B31 (2017) 1740055}

B31 (2017) 1740086

B31 (2017) 1750146

B31 (2017) 1750067

B31 (2017) 1750100

B31 (2017) 1750101

B31 (2017) 1750347

B31 (2017) 1750242

B31 (2017) 1750128

B31 (2017) 1750326 age property of industrial sectors on the global value chain

Xiong, C., Xu, W., Zhao, Y., Xiao, J. \& Zhu, X., New design graded refractive index antireflection coatings for silicon solar cells

Xiong, C., see $\mathrm{Hu}$

Xiong, P., see Hou

Xiong, X., see Wu

Xiong, Y., see Hou

$\mathrm{Xu}, \mathrm{A}$., Image system analysis of human eye wavefront aberration on the basis of HSS

Xu, B., Wang, Y. \& Ji, Z., A hierarchical energy efficiency evaluation model of numerical control workshop

Xu, B., Wang, Y. \& Ji, Z., Knowledge network model of the energy consumption in discrete manufacturing system

$\mathrm{Xu}, \mathrm{B} .-\mathrm{M} .$, see $\mathrm{Yu}$

Xu, C. \& Yang, R., Parameter estimation for chaotic systems using improved bird swarm algorithm

$\mathrm{Xu}, \mathrm{C}$., see $\mathrm{Li}$

$\mathrm{Xu}, \mathrm{C}$, see $\mathrm{Xie}$

$\mathrm{Xu}, \mathrm{C}$., see Zhao

$\mathrm{Xu}, \mathrm{D}$., see Wang

Xu, J., Jin, Y., Yuan, X. \& Miao, X., Levitation force of small clearance superconductor-magnet system under non-coaxial condition

$\mathrm{Xu}$, J., see Zhang

$\mathrm{Xu}, \mathrm{L}$., see Cong

$\mathrm{Xu}, \mathrm{L}$., see Lv

$\mathrm{Xu}, \mathrm{M}$., see Zheng

$\mathrm{Xu}, \mathrm{P}$. , see Yang

Xu, Q., Li, Y.-X., Li, X.N., Wang, J.-B., Yang, F., Yang, Y. \& Ren, T.-L., Simulation of $\mathrm{SiO}_{2}$ etching in an inductively coupled $\mathrm{CF}_{4}$ plasma

Xu, Q., Li, Y.-X., Li, X.N., Wang, J.-B., Yang, F., Yang, Y. \& Ren, T.-L.,
B31 (2017) 1750212

B31 (2017) 1740028

B31 (2017) 1740051

B31 (2017) 1750188

B31 (2017) 1750058

B31 (2017) 1750188

B31 (2017) 1740036

B31 (2017) 1740058

B31 (2017) 1740100

B31 (2017) 1750003

B31 (2017) 1750346

B31 (2017) 1750004

B31 (2017) 1750214

B31 (2017) 1740043

B31 (2017) 1750082

B31 (2017) 1750075

B31 (2017) 1750222

B31 (2017) 1750115

B31 (2017) 1740039

B31 (2017) 1740015

B31 (2017) 1740024

B31 (2017) 1750042 
Simulation and experimental verification of silicon dioxide deposition by PECVD

Xu, T., Li, M., Huang, Y., Chen, Y. \& Yu, C., Nonsingular localized wave solutions for the nonlocal Davey-Stewartson I equation with zero background

$\mathrm{Xu}, \mathrm{W}$., see Xiong

$\mathrm{Xu}, \mathrm{W}$. , see Yuan

$\mathrm{Xu}, \mathrm{X}$., see Liu

$\mathrm{Xu}, \mathrm{X}$., see Xing

$\mathrm{Xu}, \mathrm{X}$., see Zhang

$\mathrm{Xu}, \mathrm{X}$.-Y., see Li

$\mathrm{Xu}, \mathrm{Y}$., see Dai

$\mathrm{Xu}$, Y., see $\mathrm{Gu}$

$\mathrm{Xu}, \mathrm{Y}$., see Lang

$\mathrm{Xu}$, Y., see Liu

Xue, B., see Liu

Xue, G., Zhang, P., He, Z., Li, D. \& Cai, C., Structure design and driving voltage optimization of a novel giant magnetostrictive actuator

Xue, H.T., see $\mathrm{Si}$

Xue, T., see Qin

Yadav, A. \& Varshney, D., Effect of chromium substitution on crystal and lattice structure of soft manganese zinc ferrites

Yan, B., Wang, H., Yang, C. \& Wen, L., A calibration mechanism based on the principles of the Michelson interferometer microthrust test device

Yan, B.-W., see Wang

Yan, B.-W., see Wang

Yan, D., Wang, F. \& Wang, S., Research on the output bit error rate of 2DPSK signal based on stochastic resonance theory

Yan, J., see Chen

Yan, J., see Fang

Yan, K.-S., Rong, L.-L. \& Li, Q., Vulnerability analysis of interdependent spatially embedded infrastructure networks under localized attack
B31 (2017) 1750055

B31 (2017) 1750338

B31 (2017) 1740028

B31 (2017) 1750045

B31 (2017) 1740011

B31 (2017) 1750212

B31 (2017) 1730004

B31 (2017) 1750310

B31 (2017) 1750232

B31 (2017) 1750048

B31 (2017) 1740093

B31 (2017) 1750233

B31 (2017) 1750299

B31 (2017) 1750022

B31 (2017) 1750199

B31 (2017) 1750337

B31 (2017) 1750153

B31 (2017) 1750211

B31 (2017) 1750104

B31 (2017) 1750244

B31 (2017) 1850069

B31 (2017) 1750158

B31 (2017) 1750249

B31 (2017) 1750089
Yan, L., Liu, J. \& Men, S., The biospeckle method for early damage detection of fruits

Yan, L., see $\mathrm{Mu}$

Yan, W., Zuo, K. \& Deng, L., The Dirac particle in the graphene dot confined within the magnetic barriers

Yan, X.-A., Ren, B.-Q., Wang, L.-Q., Liu, Y.-W. \& Yu, H.-W., Toggle switch from optical bistability to multistability via an elliptically polarized field

Yan, X.-H. \& Lin, Y.-N., Effects of $\mathrm{Ga}$ content on the stability of Fe- $x$ $(x=6.25,12.5,18.75,25$ at.\% $\mathrm{Ga}$ ) alloys from firstprinciples calculations

Yan, X.-W., Tian, S.-F., Dong, M.-J. \& Zou, L., Characteristics of solitary waves, quasiperiodic solutions, homoclinic breather solutions and rogue waves in the generalized variable-coefficient forced Kadomtsev-Petviashvili equation

Yan, X.-W., see Dong

Yan, X.B., see Song

Yan, Z., see Dai

Yang, B., Cao, J. \& Zou, L., Moving object detection based on on-line blockrobust principal component analysis decomposition

Yang, C., see Li

Yang, C., see Yan

Yang, C., see Yao

Yang, D., see Cui

Yang, F., see $\mathrm{Xu}$

Yang, F., see $\mathrm{Xu}$

Yang, G., see Wang

Yang, G.-H., see Li

Yang, H., see Bi

Yang, H.-M., see Si

Yang, H.-W., see Yang

Yang, H.Y., Li, Q.F. \& Liu, Z.H., Electronic and optical properties of $2 \mathrm{H}$ -
B31 (2017) 1740034

B31 (2017) 1750106

B31 (2017) 1750273

B31 (2017) 1750191

B31 (2017) 1750098

B31 (2017) 1750350

B31 (2017) 1750281

B31 (2017) 1750124

B31 (2017) 1750232

B31 (2017) 1740040

B31 (2017) 1750172

B31 (2017) 1750211

B31 (2017) 1750076

B31 (2017) 1740089

B31 (2017) 1750042

B31 (2017) 1750055

B31 (2017) 1750178

B31 (2017) 1750077

B31 (2017) 1750041

B31 (2017) 1650429

B31 (2017) 1750091 
perovskite related tantalum/niobium oxides

Yang, J., see Zhang

Yang, J., see Zhang

Yang, K., see Si

Yang, L. \& Zhao, X.-F., Intelligent pulse light source in the performance calibration system of twodimensional neutron detector

Yang, L., see Li

Yang, N., Shen, K., Guo, J., Tao, X., Xu, P. \& Mao, H., Error analysis for pesticide detection performed on paper-based microfluidic chip devices

Yang, Q., see Li

Yang, R., Chai, B., Zhu, C., Wei, Q. \& Du, Z., Firstprinciples study on the structural, elastic and electronic properties of $\mathrm{Ti}_{4} \mathrm{~N}_{3}$ and $\mathrm{Ti}_{6} \mathrm{~N}_{5}$ under high pressure

Yang, R., Zhu, C., Wei, Q. \& Du, Z., Phase stability, mechanical and optoelectronic properties of two novel phases of AlN

Yang, R., see Cheng

Yang, R., see $\mathrm{Xu}$

Yang, S., see Liu

Yang, W., see Yu

Yang, W.-Y., see Zhang

Yang, X. \& Li, C., Bäcklund transformations of $Z_{n}$-sineGordon systems

Yang, X., see Chen

Yang, X., see Liu

Yang, X.-G., see Zeng

Yang, Y., Jia, L., Qin, Y., Han, S. \& Dong, H., Understanding structure of urban traffic network based on spatial-temporal correlation analysis

Yang, Y., Liu, D., Zhu, Z. \& Liu, G., Effects of $\mathrm{W}$ on hydrogen transport property of $\mathrm{Nb}_{45} \mathrm{Ti}_{27.5} \mathrm{Ni}_{27.5}$ alloy membranes Yang, Y., see Chen

Yang, Y., see Feng
B31 (2017) 1750321

B31 (2017) 1750323

B31 (2017) 1750304

B31 (2017) 1750087

B31 (2017) 1650429

B31 (2017) 1740022

B31 (2017) 1750140

B31 (2017) 1740024

B31 (2017) 1740009

B31 (2017) 1750349

B31 (2017) 1750201

B31 (2017) 1750325

B31 (2017) 1750346

B31 (2017) 1750146

B31 (2017) 1750003

B31 (2017) 1750117

B31 (2017) 1750189

B31 (2017) 1750031

B31 (2017) 1750352

B31 (2017) 1750328

B31 (2017) 1750230

B31 (2017) 1750158

B31 (2017) 1750137
Yang, Y., see Qiu

Yang, Y., see Wang

Yang, Y., see Xu

Yang, Y., see Xu

Yang, Y., see Yao

Yang, Y., see Zhao

Yang, Y., see Zhao

Yang, Z., An automatic and accurate method of full heart segmentation from CT image based on linear gradient model

Yang, Z.-J., see Gao

Yang, Z.-K., Cheng, C.-C., Wang, Z. \& Yang, H.-W., Detection of breast cancer using ultra-wide band beamforming algorithm

Yano, T., see Sun

Yao, G., Wang, H., Yang, C. \& Wen, L., Research and design of underwater flowinduced vibration energy harvester based on Karman vortex street

Yao, H., see Dong

Yao, J., see Bing

Yao, R.-H., see Liu

Yao, S., see Nian

Yao, W., Sheng, F., Wei, X., Zhang, L. \& Yang, Y., Propagation characteristics of ultrasonic guided waves in continuously welded rail

Yao, Y., Xiao, S., Zhang, X. \& Gu, X., Simulation optimizing of $n$-type HIT solar cells with AFORS-HET

Yao, Y., see Wu

Ye, F., Ye, H. \& Wang, $\mathrm{X}$, Design and experimental verification of a perfect dual-band optical metamaterial absorber

Ye, H., see Ye

Ye, J., see Cai

Ye, L., see Li

Ye, L., see Wang

Ye, Y., Di, Y. \& Song, J., Painlevé integrability and a new exact solution of a generalized HirotaSatsuma equation
B31 (2017) 1750192

B31 (2017) 1650430

B31 (2017) 1750042

B31 (2017) 1750055

B31 (2017) 1740075

B31 (2017) 1750008

B31 (2017) 1750084

B31 (2017) 1740045

B31 (2017) 1750016

B31 (2017) 1750091

B31 (2017) 1750080

B31 (2017) 1750076

B31 (2017) 1750127

B31 (2017) 1750207

B31 (2017) 1750332

B31 (2017) 1750131

B31 (2017) 1740075

B31 (2017) 1740025

B31 (2017) 1650372

B31 (2017) 1750047

B31 (2017) 1750047

B31 (2017) 1750347

B31 (2017) 1750330

B31 (2017) 1750336

B31 (2017) 1750333 
Yen, N.V., Lan, M.T., Vinh, L.T. \& Hong, N.V., Structural properties of liquid aluminosilicate with varying $\mathrm{Al}_{2} \mathrm{O}_{3} / \mathrm{SiO}_{2}$ ratios: insight from analysis and visualization of molecular dynamics data

Yi, G., see Yuan

Yi, H., see Tian

Yi, K., see Wang

Yi, S., see Zhang

Yin, A., Wang, Z. \& Fu, F., A novel semi-quantum secret sharing scheme based on Bell states

Yin, C., see Wang

Yin, H.-M., Tian, B., Zhen, H.-L., Chai, J. \& Liu, L., Pfaffian solutions for the $(3+1)$-dimensional nonlinear evolution equation in a fluid/plasma/crystal and the $(2+1)$-dimensional Sawada-Kotera equation in a liquid

Yin, H.-M., see $\mathrm{Yu}$

Yin, L., Qin, Y. \& Liu, X.-W., A new interface weak-capacitance detection ASIC of capacitive liquid level sensor in the rocket

Yin, L., see Di

Yin, L., see $\mathrm{Fu}$

Yin, L., see Li

Yin, S., see Shang

Yong, X., Fan, Y., Huang, Y., Ma, W.-X. \& Tian, J., Darboux transformation and solitons for an integrable nonautonomous nonlinear integro-differential Schrödinger equation

Yong, X., see Huang

Yong, Y., see Su

Yoshida, K., see Sun

You, F., see Zhang

Younis, M., Optical solitons in $(n+1)$ dimensions with Kerr and power law nonlinearities

Yu, C., see Xu

Yu, G.-H., Zhao, P.-Y., Xu, B.-M., Zhu, X.-L. \& Yang,
B31 (2017) 1750036
B31 (2017) 1750060
B31 (2017) 1750110
B31 (2017) 1750136
B31 (2017) 1750051

B31 (2017) 1750150 B31 (2017) 1750136

B31 (2017) 1750132 B31 (2017) 1750258

B31 (2017) 1750302
B31 (2017) 1750097
B31 (2017) 1750064
B31 (2017) 1750030
B31 (2017) 1750059

B31 (2017) 1750276

B31 (2017) 1750275

B31 (2017) 1750229

B31 (2017) 1750080

B31 (2017) 1740081

B31 (2017) 1750186
B31 (2017) 1750338
W., Isotope shift calculations of Li-like neutronrich and neutron-deficient $\mathrm{Mg}$ isotopes

Yu, H., see Zhan

Yu, H., see Zhou

Yu, H.-W., see Yan

Yu, J., see Zhang

Yu, J., see Zhao

Yu, L., see Cai

Yu, L., see Deng

Yu, L., see Liu

Yu, M.-M., Zhang, N. \& Mao, G.-Y., Comparative study on the topological structure of China Education Network

Yu, M.-X., Tian, B., Chai, J., Yin, H.-M. \& Du, Z., Bäcklund transformation, analytic soliton solutions and numerical simulation for a $(2+1)$-dimensional complex Ginzburg-Landau equation in a nonlinear fiber

Yu, S., see $\mathrm{Li}$

Yu, S.-B., see Zeng

Yu, T.-B., see Li

Yu, X.-C., see Hou

Yu, Y., see Li

Yu, Z., see Bing

Yuan, C., Wang, J. \& Yi, G., Estimation of key parameters in adaptive neuron model according to firing patterns based on improved particle swarm optimization algorithm

Yuan, F., see Ma

Yuan, F., see Teng

Yuan, H., see Zhang

Yuan, J., see Wang

Yuan, X., see Xu

Yuan, X.B., see Tian

Yuan, Y., Guo, X., An, L. \& Xu, W., Terahertz planar waveguide devices based on graphene

Yuan, Y.-Q., Tian, B., Xie, X.-Y., Chai, J. \& Liu, L., Bright-dark soliton solutions for the $(2+1)$-dimensional variable-coefficient coupled nonlinear Schrö-
B31 (2017) 1750003

B31 (2017) 1750154

B31 (2017) 1750046

B31 (2017) 1750191

B31 (2017) 1750195

B31 (2017) 1750123

B31 (2017) 1750083

B31 (2017) 1740088

B31 (2017) 1740018

B31 (2017) 1740074

B31 (2017) 1750258

B31 (2017) 1740009

B31 (2017) 1750238

B31 (2017) 1750050

B31 (2017) 1750028

B31 (2017) 1740010

B31 (2017) 1750207

B31 (2017) 1750060

B31 (2017) 1750344

B31 (2017) 1750204

B31 (2017) 1750195

B31 (2017) 1750193

B31 (2017) 1750075

B31 (2017) 1750335

B31 (2017) 1750045 
dinger system in a gradedindex waveguide

Yuan, Y.-Q., see Sun

Yue, W.W., see Tian

Yurtseven, H., Ipekoğlu, U. \& Ateş, S., Calculation of the tilt angle and susceptibility for the $\alpha-\beta$ transition in quartz using a mean field model

Yusuf, A., see Inc

Zeng, J.-W., Yang, X.-G., Qian, Y.-S. \& Wei, X.-T., Research on three-phase traffic flow modeling based on interaction range

Zeng, J.-W., Yu, S.-B., Qian, Y.-S., Wei, X.-T., Feng, X. \& Wang, H., Research on traffic flow characteristics at signal intersection

Zeng, X., see Li

Zeng, X., see Xie

Zeng, X., see Xie

Zhai, M., see Han

Zhai, W., Hu, Y.-L. \& Ran, F., CQPSO scheduling algorithm for heterogeneous multi-core DAG task model

Zhai, X.-S., see Fang

Zhan, X., Bu, X., Qin, T., Yu, H., Chen, J. \& Wei, Y., X-ray online detection for laser welding $\mathrm{T}$-joint of Al-Li alloy

Zhan, X., Chen, F., Gao, M., Tie, S. \& Gao, W., Enhanced performance of a structured cyclo olefin copolymer-based amorphous silicon solar cell

Zhan, X., see Chen

Zhan, X., see Gao

Zhan, X.Z., see Mao

Zhang, B., see $\mathrm{He}$

Zhang, B., see Sun

Zhang, B., see Zhou

Zhang, C., see Liang

Zhang, C., see Li

Zhang, C., see Tian

Zhang, C., see Zhou

Zhang, C.-J., see Li

Zhang, D., see Chao

Zhang, D., see Chen
B31 (2017) 1750100

B31 (2017) 1750065

B31 (2017) 1750335

B31 (2017) 1750092

B31 (2017) 1750163

B31 (2017) 1750328

B31 (2017) 1750238

B31 (2017) 1750164

B31 (2017) 1740055

B31 (2017) 1740086

B31 (2017) 1740097

B31 (2017) 1740050 B31 (2017) 1750194

\section{B31 (2017) 1750154}

B31 (2017) 1740003

B31 (2017) 1740001

B31 (2017) 1740002

B31 (2017) 1750197

B31 (2017) 1750240

B31 (2017) 1750218

B31 (2017) 1740082

B31 (2017) 1750260

B31 (2017) 1750085

B31 (2017) 1750259

B31 (2017) 1750190

B31 (2017) 1750050

B31 (2017) 1750311

B31 (2017) 1750086
Zhang, D., see Liu

Zhang, D.-C., see Wang

Zhang, D.-C., see Wang

Zhang, F.-P., see Fang

Zhang, G. \& Liu, H., Effect of current vehicle's interruption on traffic stability in cooperative carfollowing theory

Zhang, G., Chen, C., Lang, J., Zhao, L. \& Jiang, W., High efficiency fluorescent white OLEDs based on DOPPP

Zhang, G., see Jin

Zhang, G.-W., see Zhang

Zhang, H., Wang, J., Song, Z., Liang, J.-Q. \& Wei, L.-F., Spin-parity effect in violation of Bell's inequalities for entangled states of parallel polarization

Zhang, H., see Kuai

Zhang, H., see Liu

Zhang, H.-Q. \& Wang, Y., $N$ dark-dark solitons for the coupled higher-order nonlinear Schrödinger equations in optical fibers

Zhang, H.-Z., Jiang, R., Hu, M.-B. \& Jia, B., Analytical investigation on the minimum traffic delay at a three-phase signalized $T$ type intersection

Zhang, J., Chen, Z., Cheng, C. \& Wang, Y.X., A phase field crystal model simulation of morphology evolution and misfit dislocation generation in nanoheteroepitaxy

Zhang, J., see Bi

Zhang, J., see Deng

Zhang, J., see Li

Zhang, J., see Wang

Zhang, J., see Zhang

Zhang, J., see Zhang

Zhang, J.Y. \& Lv, J., Equilibrium geometries, electronic structure and magnetic properties of $\mathrm{Co}_{n} \mathrm{Sn}$ $(n=1-12)$ clusters from density functional calculations
B31 (2017) 1750300
B31 (2017) 1750104
B31 (2017) 1750244
B31 (2017) 1750217

B31 (2017) 1750317

B31 (2017) 1750220

B31 (2017) 1740063

B31 (2017) 1750209

B31 (2017) 1750032

B31 (2017) 1750025

B31 (2017) 1750339

B31 (2017) 1750305

B31 (2017) 1750063

B31 (2017) 1750283

B31 (2017) 1750041

B31 (2017) 1740088

B31 (2017) 1740010

B31 (2017) 1750202

B31 (2017) 1750304

B31 (2017) 1750222

B31 (2017) 1750171 
Zhang, L., Li, Y., Tang, Z., Deng, Y., Yuan, H., Yu, J. \& Zhu, X., Magnetic and electrical transport properties of $\mathrm{Sr}_{2} \mathrm{Ti}_{1-x} \mathrm{Co}_{x} \mathrm{O}_{4}$ ceramics by sol-gel

Zhang, L., Shang, J. \& Cai, G., Indium passivation impact on $\mathrm{HfO}_{2} / \mathrm{GaAs}$ interface: A first-principles study

Zhang, L., Yi, S., Hu, X., Liang, B., Zhao, W. \& Wang, Y., Synthesis and photoluminescence properties of multicolor tunable $\mathrm{GdNbO}_{4}: \mathrm{Tb}^{3+}, \mathrm{Eu}^{3+}$ phosphors based on energy transfer

Zhang, L., see Liu

Zhang, L., see Sun

Zhang, L., see Tian

Zhang, L., see Tian

Zhang, L., see Tian

Zhang, L., see Yao

Zhang, M., Gu, L., Fan, Y., Liu, Y. \& Zhou, X., Effect of temperature on DNA condensation at single molecule level

Zhang, M., Ji, Z. \& Wang, Y., Artificial bee colony algorithm with dynamic multipopulation

Zhang, M., see Chen

Zhang, M., Application of BP neural network in acoustic wave measurement system

Zhang, N., see $\mathrm{Yu}$

Zhang, P., see Xue

Zhang, Q., see He

Zhang, Q., see Tian

Zhang, Q.-Y., Hu, K.-Y. \& Yang, W.-Y., Chiralityspecific lift forces of helix under shear flows

Zhang, R., Zhou, Y., Zhu, Y., Li, Y., Chu, L., Min, Y., Zhang, J., Yang, J. \& Li, X., First principle investigations of the $\mathrm{Pbnm}$ phase $\mathrm{BiFeO}_{3}, \mathrm{BiFe}_{0.875^{-}}$ $\mathrm{Mn}_{0.125} \mathrm{O}_{3}$ and $\mathrm{Bi}_{0.875}$ $\mathrm{X}_{0.125} \mathrm{Fe}_{0.875} \mathrm{Mn}_{0.125} \mathrm{O}_{3}$ $(\mathrm{XBFM})(\mathrm{X}=\mathrm{Ce}, \mathrm{Gd}, \mathrm{Lu})$
B31 (2017) 1750195

B31 (2017) 1750203

B31 (2017) 1750051
B31 (2017) 1750266
B31 (2017) 1750218
B31 (2017) 1750110
B31 (2017) 1750259
B31 (2017) 1750014
B31 (2017) 1740075

B31 (2017) 1750147

B31 (2017) 1740087

B31 (2017) 1750242

B31 (2017) 1740052

B31 (2017) 1740074

B31 (2017) 1750022

B31 (2017) 1750213

B31 (2017) 1750267

B31 (2017) 1750117

B31 (2017) 1750304
Zhang, R., see Chen

Zhang, R., see Chu

Zhang, R., see Guo

Zhang, R., see Li

Zhang, R.-C., see Li

Zhang, R.-H., He, Z.-C., You, F. \& Chen, B., Attitude algorithm and initial alignment method for SINS applied in short range aircraft

Zhang, S.-L., see Wen

Zhang, T., Zhang, J., Xu, J., Wang, Q., Zhao, R., Liu, H., Dong, G., Hao, Y. \& Bi, $\mathrm{K}$., Wideband metasurface filter based on complementary split-ring resonators

Zhang, T., Average value of the shape and direction factor in the equation of refractive index

Zhang, W., see $\mathrm{Hu}$

Zhang, W., see Liao

Zhang, W., see Liao

Zhang, W., see Liu

Zhang, W., see Xie

Zhang, X., Choi, K.S. \& Song, L., Coherent structures induced by dielectric barrier discharge plasma actuator

Zhang, X., see Chen

Zhang, X., see $\mathrm{Hu}$

Zhang, X., see Liao

Zhang, X., see Liao

Zhang, X., see Shi

Zhang, X., see Wu

Zhang, X., see Yao

Zhang, X., see Zhang

Zhang, X.-J., see Long

Zhang, X.-X. \& Zhang, G.W., Controlling heat transport in the spin-chain system with multi-spin interaction and external field

Zhang, Y., Kang, L., Xu, X., Tang, X., Li, H. \& Cai, X., Semiclassical transport through a mesostructure and distribution of classical trajectories

Zhang, Y., see Cheng

Zhang, Y., see Sun

Zhang, Y., see Wen
B31 (2017) 1750271

B31 (2017) 1740085

B31 (2017) 1740084

B31 (2017) 1750172

B31 (2017) 1750327

B31 (2017) 1740081 B31 (2017) 1750017

B31 (2017) 1750222

B31 (2017) 1750263

B31 (2017) 1750182

B31 (2017) 1750223

B31 (2017) 1750306

B31 (2017) 1750266

B31 (2017) 1740055

B31 (2017) 1850038

B31 (2017) 1750113

B31 (2017) 1750182

B31 (2017) 1750223

B31 (2017) 1750306

B31 (2017) 1740096

B31 (2017) 1740068

B31 (2017) 1740025

B31 (2017) 1740042

B31 (2017) 1750161

B31 (2017) 1750209

B31 (2017) 1730004

B31 (2017) 1750224

B31 (2017) 1750200

B31 (2017) 1750017 
Zhang, Y., see Wu

Zhang, Y., see Zhu

Zhang, Y., Active magnetic bearing system based on sliding mode control

Zhang, Y.-H., see Hou

Zhang, Z., Fan, X. \& Zhang, $\mathrm{X}$., A fast learning based super-resolution method for copper strip defect image

Zhang, Z., Sun, B., Yang, J., Wei, Y. \& He, S., Electrostatic separation for recycling silver, silicon and polyethylene terephthalate from waste photovoltaic cells

Zhang, Z.-M., see Wang

Zhang, Z.-Y., Molecular dynamics studies on the grain growth of nanocrystalline $\mathrm{Ni}$ and $\mathrm{Ni}_{3} \mathrm{Al}$

Zhao, B., see Liu

Zhao, C., see $\mathrm{Hu}$

Zhao, D., see Li

Zhao, D.-L., see Xie

Zhao, F.Q., see Pan

Zhao, G., see Bi

Zhao, H., Li, L., Peng, H., Kurths, J., Xiao, J., Yang, Y. \& Li, A., A new approach of analyzing timevarying dynamical equation via an optimal principle

Zhao, H., Li, L., Peng, H., Xiao, J., Yang, Y. \& Zheng, M., Fixed-time synchronization of multi-links complex network

Zhao, H., Luo, X., Zhu, C., Watanabe, T. \& Zhu, T., Behavior-aware cache hierarchy optimization for low-power multi-core embedded systems

Zhao, H., Wang, G., Qi, L., Wang, L. \& Ma, L., Three-component pillared phononic crystal plate containing viscoelastic material

Zhao, H., see Ma

Zhao, H., see Teng
B31 (2017) 1750177

B31 (2017) 1750058
B31 (2017) 1740008
B31 (2017) 1740013
B31 (2017) 1750028

B31 (2017) 1740042

B31 (2017) 1750087

B31 (2017) 1750343

B31 (2017) 1750237

B31 (2017) 1750352

B31 (2017) 1750035

B31 (2017) 1750164

B31 (2017) 1750105

B31 (2017) 1750187

B31 (2017) 1740006

\section{B31 (2017) 1750084}

B31 (2017) 1750008

B31 (2017) 1740067

B31 (2017) 1750344

B31 (2017) 1750204
Zhao, H., see Zhu

Zhao, H., Iterative-decreasing calibration method based on regional circle

Zhao, J., Guo, J., Cheng, W., Xu, C. \& Huang, L., Assessment of SPOT-6 optical remote sensing data against GF-1 using NNDiffuse image fusion algorithm

Zhao, J., see Dong

Zhao, J., see Gao

Zhao, J.-C., see Zhu

Zhao, L., see Sun

Zhao, L., see Zhang

Zhao, P.-Y., see Yu

Zhao, R., see Zhang

Zhao, W., Han, X., Li, R., Zheng, Y. \& Wang, Y., Effects of size and concentration of silt particles on flow and performance of a centrifugal pump under cavitating conditions

Zhao, W., Su, Y. \& Wang, D., Synergetic effects of interfacial and spatial confinement in polymer nanocomposites

Zhao, W., see Liu

Zhao, W., see Zhang

Zhao, X., see Gong

Zhao, X., see Li

Zhao, X.-F., see Lin

Zhao, X.-F., see Yang

Zhao, X.-H., Tian, B., Liu, D.-Y., Wu, X.-Y., Chai, J. \& Guo, Y.-J., Dark solitons, Lax pair and infinitely-many conservation laws for a generalized $(2+1)$-dimensional variable-coefficient nonlinear Schrödinger equation in the inhomogeneous Heisenberg ferromagnetic spin chain

Zhao, X.-J. \& Hao, H.-Q., $n$-fold Darboux transformation, conservation laws and modulation instability for the semi-discrete coupled nonlinear Schrödinger equation
B31 (2017) 1740061

B31 (2017) 1740031

B31 (2017) 1740043

B31 (2017) 1750127

B31 (2017) 1750070

B31 (2017) 1740062

B31 (2017) 1750218

B31 (2017) 1750220

B31 (2017) 1750003

B31 (2017) 1750222

B31 (2017) 1750312

B31 (2017) 1730003

B31 (2017) 1750266

B31 (2017) 1750051

B31 (2017) 1750286

B31 (2017) 1750148

B31 (2017) 1740047

B31 (2017) 1740022

B31 (2017) 1750013

B31 (2017) 1750174 
Zhao, X.-M., Yu, J., He, J., Cheng, Q.-B., Liang, Y. \& Kou, S.-P., The simulation of non-Abelian statistics of Majorana fermions in Ising chain with Z2 symmetry

Zhao, Y., see Liao

Zhao, Y., see Liao

Zhao, Y., see Liu

Zhao, Y., see $\mathrm{Li}$

Zhao, Y., see Xiong

Zhao, Z., Chen, Y. \& Han, B., Lump soliton, mixed lump stripe and periodic lump solutions of a $(2+1)$ dimensional asymmetrical Nizhnik-Novikov-Veselov equation

Zhao, Z., see Chen

Zhen, H.-L., see Chai

Zhen, H.-L., see Yin

Zhen, Y. \& Zhou, L., Wave propagation in fluid-conveying viscoelastic carbon nanotubes under longitudinal magnetic field with thermal and surface effect via nonlocal strain gradient theory

Zhen, Z. \& He, J., Temperature diagnostics of plasma under optically-thin conditions

Zheng, B.-H., Material procedure quality forecast based on genetic BP neural network

Zheng, L., Shi, H. \& Gu, M., Infrared traffic image enhancement algorithm based on dark channel prior and gamma correction

Zheng, M., see Tian

Zheng, M., see Tian

Zheng, M., see Tian

Zheng, M., see Zhao

Zheng, Q., see Li

Zheng, T., see $\mathrm{Li}$

Zheng, Y., Chen, X. \& Zhu, R., Frequency hopping signals detection based on wavelet decomposition and Hilbert-Huang transform

Zheng, Y., see Sun
B31 (2017) 1750123
B31 (2017) 1750223
B31 (2017) 1750306
B31 (2017) 1750073
B31 (2017) 1740010
B31 (2017) 1740028

B31 (2017) 1750157

B31 (2017) 1750034

B31 (2017) 1750135

B31 (2017) 1750132

B31 (2017) 1750069

B31 (2017) 1750292

B31 (2017) 1740080

B31 (2017) 1740044

B31 (2017) 1750110

B31 (2017) 1750259

B31 (2017) 1750014

B31 (2017) 1750008

B31 (2017) 1750326

B31 (2017) 1750090

B31 (2017) 1740078 B31 (2017) 1750218
Zheng, Y., see Zhao

B31 (2017) 1750312

Zheng, Z. \& Xu, M., Active magnetic levitation guide based on magnetic damping control

Zheng, Z.-Q., see Zhu

Zheng, Z.-Q., see Zhu

Zhi, M., Tang, L. \& Qiao, D., Design and analysis of miniature tri-axial fluxgate magnetometer

Zhon, K., see Xie

Zhong, Z.-X., see Qi

Zhou, B., see Chen

Zhou, C., see Cheng

Zhou, C.-F., see Wang

Zhou, C.-F., see Wang

Zhou, C.-F., see Wang

Zhou, C.-F., see Wang

Zhou, H.-B., see Li

Zhou, J., see Han

Zhou, J., see Liu

Zhou, J., see Wang

Zhou, L., see Zhen

Zhou, R., see Liu

Zhou, R.-G., Tan, C. \& Fan, P., Quantum multidimensional color image scaling using nearest-neighbor interpolation based on the extension of FRQI

Zhou, S., Bai, M. \& Zhang, C., Analysis and construction of four-party deterministic operation sharing with a generalized sevenqubit Brown state

Zhou, W., Wang, D., Yu, H. \& Peng, B., A pressuredeformation analytical model for rectangular diaphragm of MEMS pressure sensors

Zhou, X., Jun, S., Zhang, B. \& Jun, W., Classification of different kinds of pesticide residues on lettuce based on fluorescence spectra and WTBCC-SVM algorithm

Zhou, X., Wang, J., Wang, R. \& Lin, J., Band gaps in grid structure with periodic local resonator subsystems

Zhou, X., see Zhang
B31 (2017) 1740015

B31 (2017) 1740012

B31 (2017) 1740014

B31 (2017) 1750040

B31 (2017) 1750105

B31 (2017) 1750319

B31 (2017) 1740065

B31 (2017) 1750325

B31 (2017) 1750104

B31 (2017) 1750244

B31 (2017) 1750291

B31 (2017) 1750353

B31 (2017) 1750002

B31 (2017) 1740097

B31 (2017) 1750146

B31 (2017) 1750343

B31 (2017) 1750069

B31 (2017) 1750146

B31 (2017) 1750184

B31 (2017) 1750190

B31 (2017) 1750046

B31 (2017) 1740082

B31 (2017) 1750225
B31 (2017) 1750147 
Zhou, Y., see Zhang

Zhou, Z., see Zhu

Zhu, C., Du, J.-M., Zhao, J.C., Zhu, T. \& Chen, G.Q., Molecular and structural characterization of New Red and Erythrosine by fluorescence polarization spectroscopy

Zhu, C., Zhao, H., Chen, T. \& Zhu, T., A low latency and high efficient three-dimension Networkon-Chip based on hierarchical structure

Zhu, C., see Yang

Zhu, C., see Yang

Zhu, C., see Zhao

Zhu, G., Ruan, X. \& Zhou, Z., Dispersion characteristics and compensation in the POLMUX coherent optical communication system

Zhu, G., see Tian

Zhu, H.-B., see Wang

Zhu, H.-B., see Wang

Zhu, J.-M., see Li

Zhu, J.-R., see Leng

Zhu, L. \& Li, J., Phase separation of two-component Bose-Einstein condensates with monopolar interaction

Zhu, M., see Wang

Zhu, R., Chen, X. \& Huang, $\mathrm{Y}$., The sequence relay selection strategy based on stochastic dynamic programming

Zhu, R., see Zheng

Zhu, T., Zhang, Y. \& Ji, R., Design of a charge pump for high voltage driver applications based on 0.35 $\mu \mathrm{m}$ BCD technology

Zhu, T., see Zhao

Zhu, T., see Zhu

Zhu, T., see Zhu

Zhu, X., Xiang, R., Wu, F. \& Jiang, X., Single image haze removal based on fusion darkness channel prior

Zhu, X., see Chen

B31 (2017) 1750304
B31 (2017) 1740054


B31 (2017) 1740062

B31 (2017) 1740061
B31 (2017) 1750201
B31 (2017) 1750349
B31 (2017) 1740067

B31 (2017) 1740054

B31 (2017) 1750267

B31 (2017) 1750291

B31 (2017) 1750353

B31 (2017) 1750296

B31 (2017) 1740053

B31 (2017) 1750215

B31 (2017) 1750136

B31 (2017) 1740076

B31 (2017) 1740078

B31 (2017) 1740008

B31 (2017) 1740067

B31 (2017) 1740061

B31 (2017) 1740062

B31 (2017) 1740037

B31 (2017) 1740016
Zhu, X., see Jin

Zhu, X., see Wu

Zhu, X., see Xiang

Zhu, X., see Xiong

Zhu, X., see Zhang

Zhu, X.-F., see Chen

Zhu, X.-F., see Li

Zhu, X.-F., see Rao

Zhu, X.-L., see Yu

Zhu, Y. \& Guan, H., Optical communication equalized technique suitable for high-speed transmission

Zhu, Y., Tian, L. \& Wan, T., Automatic polygon mesh repair and simplification for three-dimensional human modeling

Zhu, Y., Wei, S.-C., Dong, Y.C., Liang, Y. \& Wang, Y.J., The study of a novel ultrasonic A-scan signal processing method based on fractal theory

Zhu, Y., see Hou

Zhu, Y., see Zhang

Zhu, Y.-B., see Cai

Zhu, Y.-L. \& Zheng, Z.-Q., Dynamic responses of the rotor supported by a new type zero-clearance catcher bearing

Zhu, Y.-L. \& Zheng, Z.-Q., The use of double-decker catcher bearing with faceto-face installed inner layer bearings

Zhu, Z., see Yang

Zia, M.F., see AbdelRahman

Zou, L., Tian, S.-F. \& Feng, L.-L., Nonlocal symmetries, solitary waves and cnoidal periodic waves of the $(2+1)$-dimensional breaking soliton equation

Zou, L., see Chen

Zou, L., see Dong

Zou, L., see Yang

Zou, L., see Yan

Zou, Q., Mo, S., Wang, Y., Dang, M., Qin, G., Fu, X., Wang, H. \& Tao, X., Assembly-line flash synthesis of $\mathrm{ZnO}$ nanobelts on
B31 (2017) 1740063
B31 (2017) 1740046
B31 (2017) 1740038
B31 (2017) 1740028
B31 (2017) 1750195
B31 (2017) 1740079
B31 (2017) 1740049
B31 (2017) 1740019
B31 (2017) 1750003

B31 (2017) 1740048

B31 (2017) 1740077

B31 (2017) 1740027

B31 (2017) 1750251

B31 (2017) 1750304

B31 (2017) 1750083

B31 (2017) 1740014

B31 (2017) 1740012

B31 (2017) 1750321

B31 (2017) 1750145

B31 (2017) 1750348

B31 (2017) 1740065

B31 (2017) 1750281

B31 (2017) 1740040

B31 (2017) 1750350 
Author Index

metal Zn

Zou, Q., see Qin

Zou, W., see Gong

Zou, X., see Mu

Zou, X., see Shi

Zou, X.-Y., Simulation of $\mathrm{n}-\beta-\mathrm{FeSi}_{2} / \mathrm{p}$-Si heterojunction solar cells based on AFORS-HET

Zou, Y. \& Quan, L., A new service-oriented gridbased method for AIoT ap-
B31 (2017) 1750250

B31 (2017) 1750337

B31 (2017) 1750286

B31 (2017) 1750106

B31 (2017) 1740096

B31 (2017) 1740026 plication and implementation

Zou, Y. \& Quan, L., Resource management and scheduling policy based on grid for AIoT

Zou, Y.-Y. \& Hu, Y., An approach of traffic signal control based on NLRSQP algorithm

B31 (2017) 1750293

Zu, X.D., see Ma

Zuo, K., see Yan
B31 (2017) 1740064

B31 (2017) 1740066

B31 (2017) 1750018

B31 (2017) 1750273 